\title{
ARTICLE
}

\section{Irregularities and Roughness}

\section{G.T. Michaltsos* T. Avraam}

National Technical University of Athens, Athens, Greece

\begin{tabular}{l}
\hline ARTICLE INFO \\
\hline Article history \\
Received: 3 June 2021 \\
Accepted: 13 July 2021 \\
Published Online: 20 July 2021 \\
\hline Keywords: \\
Irregularities \\
Roughness \\
Bridges dynamics \\
Vehicle models
\end{tabular}

\section{Introduction}

The influence of a rough deck-surface on the dynamic response of a bridge depends on various factors. Irregularities, on a deck-surface, may arise due to the roughness of the deck-surface, or also due to constructed pavement anomalies for traffic reasons.

The parameter of the road surface (or bridge deck) roughness, is dealt with excessively in recent literature.

In 1960, Carey and Irick ${ }^{[1]}$ showed that surface roughness was the primary variable needed to explain the driver's opinion about the quality of the serviceability provided by a pavement surface. Most of the investigations that followed focused on the study, characterization and classification of the pavements ${ }^{[2-6]}$.

Numerous studies adopt the Power Spectral Density (PSD) functions for roughness, as modified by Wang and Huang ${ }^{[7]}$, or the simpler harmonically varying surface irregularity presented in Cheng et al ${ }^{[8]}$.

*Corresponding Author:

G.T. Michaltsos,

National Technical University of Athens, Athens, Greece;

Email: michalts@central.ntua.gr

\begin{abstract}
The periodically repeated pavement irregularities and their effect on the dynamic behavior of a bridge are the subject of this paper, as well as a new point of view of how the surface roughness operates on vehicles. The authors observed that the models used so far accept that the wheels are always in contact with the roughness curve. But in reality the wheels only come in contact with the peaks of the roughness curve by applying impact forces. The theoretical formulation is based on a continuous approach that has been used in literature to analyze such bridge. The procedure is carried out by the modal superposition method, while the obtained equations are solved by using Duhamel's integrals. Important conclusions for structural design purposes can be drawn through a variety of numerical examples.
\end{abstract}

Among these, significant contributions by Fatard et al ${ }^{[9]}$, Chang and Lee ${ }^{[10]}$, Huang and Wang ${ }^{[11]}$, Kou and DeWolf ${ }^{[12]}$ as well as by Yang et al ${ }^{[13]}$, must be quoted.

Their findings have shown that the foregoing parameter is one of the most important factors affecting elastic dynamic response, especially applicable to steel highway bridges.

Most recent researches have focused on two main subjects.

A number of researchers study the noise and inconvenience caused by pavement roughness ${ }^{[14,15]}$, or the percentage influence of roughness on the vehicle-structure coupled interaction ${ }^{[16,17]}$. Other researchers try to determine the bridges' frequencies by studying the influence of the road surface roughness on a moving vehicle or on a pair of vehicles ${ }^{[18-20]}$.

Regarding the deck's irregularities we can classify them into individual and periodical repeats. The individual ones may have been structured to reduce the speed of the 
cars as they enter a bridge or for other traffic reasons. The form, the correct position and the lower and upper critical speeds (which are unique for each irregularity as a vehicle passes it) have been studied in detail ${ }^{[21,22]}$.

Periodically repeated irregularities and their effect on the dynamic behavior of a bridge are the subject of this paper, as well as a new point of view of how the surface roughness operates on vehicles. The theoretical formulation is based on a continuous approach that has been used in literature to analyze such bridge. The procedure is carried out by the modal superposition method and the obtained equa-tions are solved by using Duhamel's integrals.

A variety of numerical examples allow to draw important conclusions for structural design purposes.

\section{Introductory Concepts}

\subsection{Irregularities}

The irregularities have length ranging from 0.20 to 3 or 5 meters. Their usual form is shown in Figure 1a for an individual case, while Figure $1 \mathrm{~b}$ refers to periodically repeated irregularities.
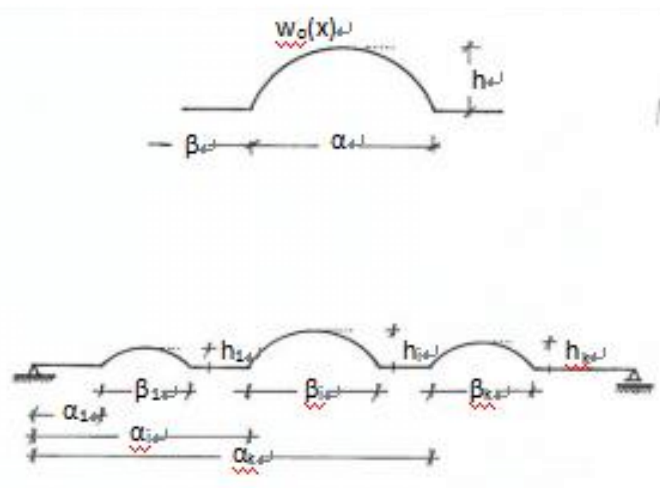

Figure 1. Typical series of irregularity

For easier mathematical manipulation, we suppose that the form of the deck is expressed for $\lambda$ repeated irregularities by the relation of equation 1 :

$$
w_{o}(x)=\sum_{k=1}^{\lambda} h_{k} \cdot \sin \frac{\pi\left(x-\alpha_{k}\right)}{\beta_{k}} \cdot\left[H\left(x-\alpha_{k}\right)-H\left(x-\alpha_{k}-\right.\right.
$$$$
\left.\beta_{k}\right) \text { ] }
$$

\subsection{Roughness}

Road roughness is characterized by the quality of the pavement.

Unlike the irregularities, the roughness has dimensions ranging from 0.2 to $5 \mathrm{~cm}$, and consists of many small scattered irregularities with depth, usually, less than $1 \mathrm{~cm}$.

According to ISO-8608 and 4287 standards, road surface roughness is associated with vehicle speed, using a formula that defines the relationship between the speed and displacement of the PSD function (Power Spectra Density).

The most general form of the PSD displacement function or road roughness is expressed in equations ( $2 \mathrm{a}$ and $b)$ :

$$
\begin{aligned}
& G_{d}\left(n_{s}\right)=G_{d}\left(n_{o}\right)\left(\frac{n_{s}}{n_{o}}\right)^{-a}\left(n_{o}=0.1 \text { cycle } / \mathrm{m}\right) \\
& G_{d}\left(n_{s}\right)=G_{d}\left(n_{o}\right)\left(\frac{\Omega_{s}}{\Omega_{o}}\right)^{-a}\left(\Omega_{o}=1 \mathrm{rad} / \mathrm{sec}\right)
\end{aligned}
$$

where $n_{o}$ is the reference spatial frequency, taken to be $0.1 \mathrm{cycle} / \mathrm{m}$. The exponent $\alpha$ is taken as 2 for a vehicle moving at constant speed. $G_{d}\left(n_{o}\right)$ is found using the values of table 3 of ISO-8608.

The road roughness function is given in equation (3) and it is obtained by applying the inverse Fourier transform to equations $(2 \mathrm{a}, \mathrm{b})$.

$$
w_{o}(x)=\sum_{i=1}^{N} \sqrt{4 G_{d}\left(n_{s, i}\right) \cdot \Delta_{n}} \cdot \cos \left(2 \pi n_{s, i} \cdot x+\theta_{i}\right)
$$

where $\theta_{i}$ refers to randomly selected phase angles with normal distribution between 0 and $2 \pi$. The coefficient $\Delta_{n}$ is determined by the relation $\Delta_{n}=\left(n \min _{\max }\right.$, where $\mathrm{N}$ is the total number of frequency steps between $\operatorname{nmin}_{\max }$.
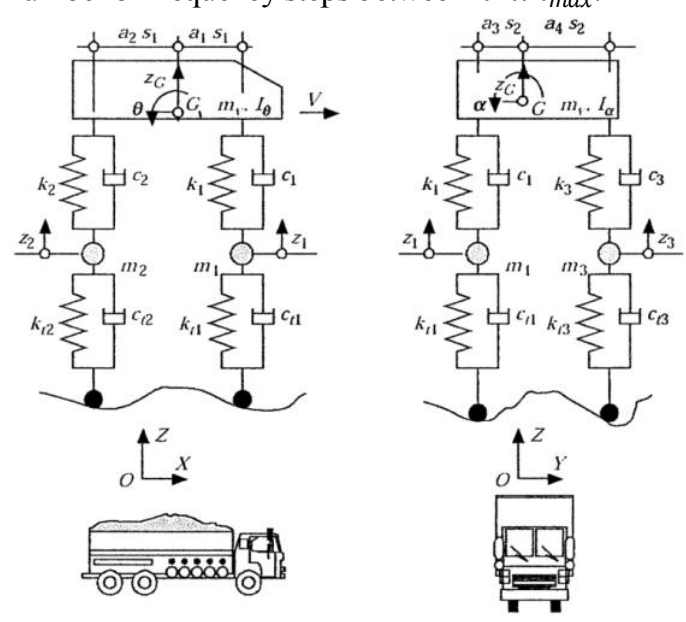

Figure 2. Vehicle's model for roughness

The above function $\mathrm{w}(\mathrm{x})$ is introduced into the equations of the vehicle's motion (see the usual model of Figure 2), in order to proceed with the analysis. The wheels of the vehicle are considered to be moving along the surface given by equation (3).

The above consideration (namely that the vehicle follows the whole curve of the irregularity since the wheels are modeled as a point), is the main deficiency of the method as the real wheel dimensions do not allow the above model for roughness ranging from 0.2 to $10 \mathrm{~cm}$.

\subsection{Loading and Bridge Models}

In this study, we use three kinds of moving loads or vehicles. 
The one actual load, is an idealized mass M, moving with constant speed $v$ (Figure 3 model 1).

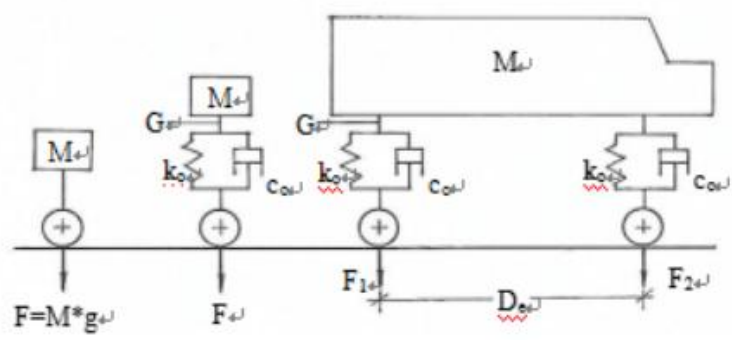

Model 1 Model 2 Model 3

Figure 3. Kinds of moving loads

The second actual load, is an idealized mass $\mathrm{M}$, moving with constant speed $v$, on a spring of constant $\mathrm{k}_{\mathrm{o}}$ and on a damper of constant $c_{o}$ as it is shown in Figure 3 Model 2. Finally the vehicle's model used, is shown in Figure 3 Model 3.

We assume also that the wheels are always in contact with the deck surface of the bridge while the speed $v$ is lower of both critical ones as they are determined in ${ }^{[22]}$.

The studied bridges are of one span with length L, while are made of homogeneous and isotropic material with modulus of elasticity $\mathrm{E}$, mass per unit length $\mathrm{m}$ and moment of inertia $I_{y}$. The damping coefficient $c_{b}$ is considered to be constant along the bridge.

\section{Moving Damped Mass-load}

\subsection{Irregularities}

Because of the limited length of such an irregularity (compared to the bridge length), it can be assumed that the velocity $v$ of the load remains constant during crossing the irregularity.

We consider that the form of the irregularity of Figure 4 is given by the following equation:

$w_{o}=w_{o}(x)$

The above described moving mass $M$ (where $M$ does not include the mass of the wheels $m_{0}$ ) passes from the beginning of the irregularity at the time $t=x_{o} / v$. Before the mass $\mathrm{M}$ enters the bridge, it moves on the horizontal axis (a), from which we will measure the movements $w$ (see Figure 4).

Under the above assumptions and considerations, one can establish the governing differential equations of the motion for a slender beam. This equation, after neglecting the effect of longitudinal motion, and using the Dirac delta function is given by the following equation:

$$
E I_{y} w^{\prime \prime \prime \prime}(x, t)+c_{b} \dot{w}(x, t)+m \ddot{w}(x, t)=F \cdot \delta(x-\alpha)+
$$$$
P_{\text {imp }} \delta(x-\alpha)
$$

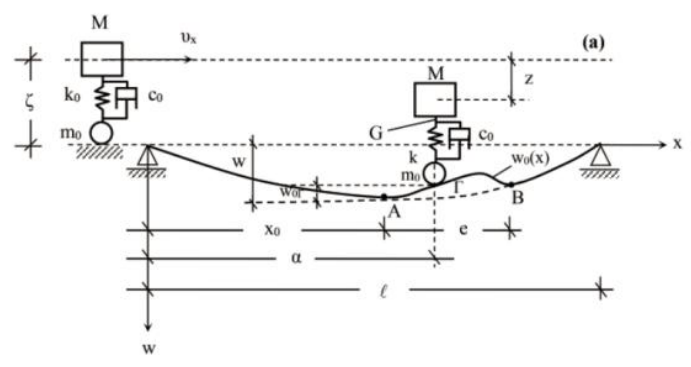

Figure 4. Irregularity on the bridge

where $P_{i m p}$ is the impact force that appears when the load enters the irregularity, that is, when the tangent to the irregularity at point A (see Figure 4) becomes different from the tangent of the beam. The associate boundary and initial conditions are respectively:

$$
\left.\begin{array}{c}
w(0, t)=w(L, t)-w^{\prime \prime}(0, t)=w^{\prime \prime}(L, t)=0 \\
w(x, 0)=\dot{w}(x, 0)=0
\end{array}\right\}
$$

From Figure 4 we find that load $\mathrm{F}$ is equal to:

$F=M(g-\ddot{z})+m_{o}\left[g-\left(\ddot{w}-\ddot{w}_{o}\right)=M(g-\ddot{z})+m_{o}(g-\ddot{w})(6 \mathrm{a})\right.$

On the other hand, cutting at point $G$ (Figure 4) and taking into account the equilibrium of forces we get: $M \ddot{z}=-k_{o}\left[z-\left(w-w_{o}\right)\right]-c_{o}\left[\dot{z}-\left(\dot{w}-\dot{w}_{o}\right)\right]=-k_{o} z+$ $k_{o} w-k_{o} w_{o}-c_{o} \dot{z}+c_{o} \dot{w}$, which leads to:

$\ddot{z}+2 \beta_{p} \dot{z}+\omega_{p}^{2} z=\Phi(t)-\omega_{p}^{2} w_{o}(x)$

where:

$\Phi(t)=\omega_{p}^{2} w(x, t)+2 \beta_{p} \dot{w}(x, t)$

with: $\left.\beta_{p}=c_{o} / 2 M, \omega_{p}^{2}=k_{o} / M\right\}$

In addition we have (see Figure 1):

$w_{o}=0$ for $\left.\alpha_{i}+\beta_{i} \leq x \leq \alpha_{i+1}\right\}$ $w_{o}=w_{o}(x)$ " $\left.\alpha_{i} \leq x \leq \alpha_{i}+\beta_{i}\right\}$

The solution of equation (6b), with initial conditions $z(0)=\dot{z}(0)=0$, is given by the Duhamel's integral:

$$
\left.\begin{array}{c}
z(0)=\frac{1}{\bar{\omega}_{p}} \cdot \int_{0}^{t} e^{-\beta} p(t-\tau)\left[\phi(\tau)-\omega_{p}^{2} w_{0}(x)\right] \sin \bar{\omega}_{p}(t-\tau) d \tau \\
\text { where }: \bar{\omega}_{p}=\sqrt{\omega_{p}^{2}-\beta_{p}^{2}}
\end{array}\right\}
$$

The Leibnitz's formula, for $G(x)=\int_{0}^{x} Q(x, t) d t$, gives: $\frac{d G(x)}{d x}=Q(x, x)+\int_{0}^{x} \frac{\partial Q(x, t)}{\partial x} d t$.

Because of the above, equation (7a) gives successively: $\dot{z}(t)=\frac{1}{\bar{\omega}_{p}} \cdot \int_{0}^{t} e^{-\beta_{p}(t-\tau)}\left[\Phi(\tau)-\omega_{p}^{2} w_{o}(x)\right] \cdot\left[\bar{\omega}_{p} \cos \omega_{p}(t-\right.$

$\left.\tau)-\beta_{p} \sin \bar{\omega}_{p}(t-\tau)\right] d \tau$ and finally:

$$
\left.\begin{array}{c}
\ddot{z}(t)=\Phi(t)-\omega_{p}^{2} w_{o}(x)+ \\
\frac{1}{\bar{\omega}_{p}} \int_{0}^{t} e^{-\beta_{p}(t-\tau)}\left[\Phi(\tau)-\omega_{p}^{2} w_{o}(x)\right] \cdot\left[\left(\beta_{p}^{2}-\bar{\omega}_{p}^{2}\right) \sin \bar{\omega}_{p}\right. \\
(t-\tau)-2 \beta_{p} \bar{\omega}_{p} \cos \omega_{p}(t-\tau) d \tau
\end{array}\right\}
$$


Because of (6a), the bridge's equation of motion becomes:

$E I_{y} w^{\prime \prime \prime}+c_{b} \dot{w}+m \ddot{w}=\left[M(g-\ddot{z})+m_{o}(g-\ddot{w})\right] \delta(x-$

$\alpha)+P_{i m p} \delta\left(x-\alpha_{i}-\xi_{o}\right) \delta\left(t-t_{\alpha i}\right.$

The impact load inserted in the above equation (8), is equal to (see Appendix B):

$P_{i m p}=N \sin \phi=\frac{\varepsilon m_{o} v}{2} \cdot \sin \phi$

where $\varphi$ is the angle shown in Figure 5.

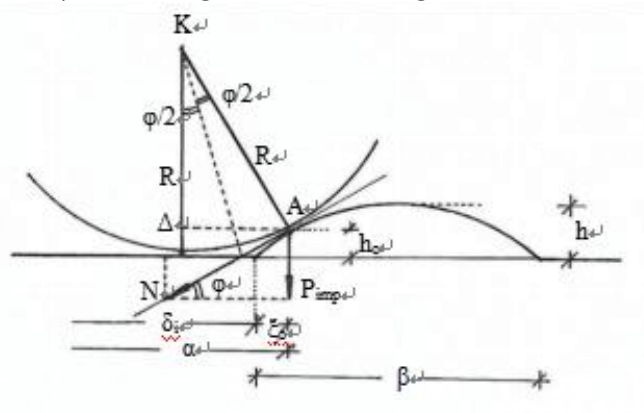

Figure 5. The developed impact force

In order to determine angle $\varphi$, we need to determine the point A i.e. the $\xi_{0}$. From equation (1) we get $w_{o}(x)=h$. $\sin \frac{\pi x}{\beta}$ and therefore:

$\tan \phi=w_{o}^{\prime}(x)=\frac{\pi h}{\beta} \cdot \cos \frac{\pi \xi_{o}}{\beta}$

From Figure 5 we obtain: $A \Delta=\sqrt{R^{2}-\left(R-w_{o}\left(\xi_{o}\right)\right)^{2}}, K \Delta=$ $R-w_{o}\left(\xi_{o}\right), \tan \phi=w_{o}^{\prime}\left(\xi_{o}\right)=\frac{A \Delta}{K \Delta}$

which concludes to the following equation:

$w_{o}^{\prime}\left(\xi_{o}\right) \cdot\left[R-w_{o}\left(\xi_{o}\right)\right]=\sqrt{2 R w_{o}\left(\xi_{o}\right)-w_{o}^{2}\left(\xi_{o}\right)}$

Solving the above arithmetically one can determine $\xi_{\mathrm{o}}$, and finally:

$\tan \phi=\frac{\pi h}{\beta} \cdot \cos \frac{\pi \xi_{o}}{\beta}$

In order to solve equation (8), we are searching for a solution under the form:

$w(x, t)=\sum_{n} X_{n}(x) \cdot T_{n}(t)$

where $X_{n}(x)$ are the shape functions of the beam and $T_{n}(t)$ are the time functions to be determined. Introducing $w(x, t)$ from equation (10) into equation (8) and taking into account equation (9a), we obtain the following equations:

$$
\left.\begin{array}{c}
E I_{y} \sum_{n} X_{n}^{\prime \prime \prime} T_{n}+c_{b} \sum_{n} X_{n} \dot{T}_{n}+m \sum_{n} X_{n} \ddot{T}_{n}= \\
\left.+\left(M+m_{o}\right) g-M \ddot{z}-m_{o} \sum_{n} X_{n} \ddot{T}_{n}\right] \delta(x-\alpha) \\
+\frac{\varepsilon m_{o} v}{2} \cdot \sin \phi \sum_{i=1}^{\zeta} \delta(x-\alpha) \delta\left(t-t_{a_{i}}\right)
\end{array}\right\}
$$

Where:

$$
\begin{gathered}
\ddot{z}=\Phi(t)-\omega_{p}^{2} w_{o}(x)+\frac{1}{\bar{\omega}_{p}} \int_{0}^{t} \Xi(\tau)\left[\Phi(\tau)-\omega_{p}^{2} w_{o}(x)\right] d \tau \\
\Phi(t)=\sum_{n} \sin \Omega_{n} t\left(\omega_{p}^{2} T_{n}+2 \beta_{p} \dot{T}_{n}\right) \\
\Xi(t)=e^{-\beta_{p}(t-\tau)}\left[\left(\beta_{p}^{2}-\omega_{p}^{2}\right) \sin \omega_{p}(t-\tau)+\right. \\
\left.2 \beta_{p} \bar{\omega}_{p} \cos \omega_{p}(t-\tau)\right] \\
\Omega_{n}=\frac{n \pi v}{L}, \quad t_{\alpha_{i}}=\frac{\alpha_{i}+\xi_{o}}{v}, \quad \alpha=\alpha_{i}+\xi_{o}
\end{gathered}
$$

and $\zeta$ the number of irregularities (see also Figure 1).

The free vibrating beam is governed by the equation $E I_{y} X_{n}^{\prime \prime \prime}-m \omega_{n}^{2} X_{n}=0$, where, due to the conditions of a simply supported one span beam, we have $X_{n}=$ $\sin \frac{n \pi x}{L}, \quad \omega_{n}^{2}=\frac{n^{4} \pi^{4} E I_{y}}{m L^{4}}$.

Because of the above, equation (11a) becomes: $m \sum_{n} X_{n} \ddot{T}_{n}+c_{b} \sum_{n} X_{n} \dot{T}_{n}+m \sum_{n} \omega_{n}^{2} X_{n} T_{n}=[(M+)$

$$
\left.\begin{array}{c}
\left.\left.m_{o}\right) g-M \ddot{z}-m_{o} \sum_{n} X_{n} \ddot{T}_{n}\right] \delta(x-\alpha) \\
+\frac{\varepsilon m_{o} v}{2} \cdot \sin \phi \sum_{i=1}^{\zeta} \delta(x-\alpha) \delta\left(t-t_{a_{i}}\right)
\end{array}\right\}
$$

Multiplication of equation (11c) by $X_{k}(x), \quad(k \neq n)$ and integration of resultant quantities from 0 to $\mathrm{L}$, leads to the following equation:

$$
\left.\begin{array}{c}
\ddot{T}_{k}+\frac{c_{b}}{m} \dot{T}_{k}+\omega_{k}^{2} T_{k}=\left[\left(M+m_{o}\right) g-M Z(t)-m_{o}\right. \\
\left.\sum_{n} X_{n} \ddot{T}_{n}\right] \sin \Omega_{k} t \\
+\frac{\varepsilon m_{o} v}{m L} \cdot \sin \phi \sum_{i=1}^{\zeta} \sin \frac{k \pi \alpha}{L} \cdot \delta\left(t-t_{a_{i}}\right) \\
\text { where }: Z(t)=\Phi(t)-\omega_{p}^{2} w_{o}(\alpha)+\frac{1}{\bar{\omega}_{p}} \\
\int_{0}^{t} \Xi(\tau)\left[\Phi(\tau)-\omega_{p} w_{o}(\alpha)\right] d \tau
\end{array}\right\}
$$

Clearly, a closed form solution of equation (11d) is not possible. However, one can seek approximate solutions, based on previous pertinent works ${ }^{[2,23]}$.

A first approximate solution of equation (11d) is obtained by considering as loading the force $P=M \cdot g$ and by ignoring the irregularities existence. This leads to $\bar{T}_{k}=\frac{2 M g}{m L\left(\omega_{k}^{2}-\Omega_{k}^{2}\right)} \cdot\left(\sin \Omega_{k} t-\frac{\Omega_{k}}{\omega_{k}} \sin \omega_{k} t\right)$, with $\left.\Omega_{k}=\frac{k \pi v}{L}\right\}(11 \mathrm{e})$

Introducing the last expression into the right-hand side of equation (11d), we obtain:

$$
\left.\begin{array}{c}
\ddot{T}_{k}+\frac{c_{b}}{m} \dot{T}_{k}+\omega_{k}^{2} T_{k}=\left[\left(M+m_{o}\right) g-M Z(t)\right. \\
\left.-m_{o} \sum_{n} X_{n} \ddot{\bar{T}}_{n}\right] \sin \Omega_{k} t \\
+\frac{\varepsilon m_{o} v}{m L} \cdot \sin \phi \sum_{i=1}^{\zeta} \sin \frac{k \pi \alpha}{L} \cdot \delta\left(t-t_{a_{i}}\right) \\
\text { with: } Z(t) \text { from }(11 d) \text { and } \Phi(t)= \\
\sum_{n} \sin \Omega_{n} t\left[\omega_{p}^{2} \bar{T}_{n}+2 \beta_{p} \dot{\bar{T}}_{n}\right]
\end{array}\right\}
$$

The solution of equation (12a), due to the initial conditions ( $5 \mathrm{~b})$, is given by the Duhamel's integral:

$$
\left.\begin{array}{c}
T_{k}=\frac{2}{m L \bar{\omega}_{k}} \int_{0}^{t}\left[\left(M+m_{o}\right) g-M Z(\tau)-m_{o} \sum_{n} \ddot{\bar{T}}_{n}(\tau) \sin \Omega_{n} t\right] \\
\sin \Omega_{n} \tau \cdot e^{-\beta(t-\tau)} \sin \omega k(t-\tau) d \tau \\
+\frac{\varepsilon m_{o} v}{m L \bar{\omega}_{k}} \sum_{i=1}^{\zeta} \sin \phi_{i} \sin \Omega_{k} t_{a} \cdot e^{-\beta(t-\tau)} \sin \omega_{k}\left(t-t_{a}\right) \\
{\left[H\left(t--_{v}^{a_{i}}\right)-H\left(t-a_{v}^{a_{i}+\beta_{i}}\right)\right]}
\end{array}\right\}
$$

with $t_{a}$ from (11b) and $\mathrm{H}$ the unit step function.

\subsection{Roughness}

In $\$ 2.2$ we noted that the wheel of a vehicle cannot follow the entire surface of the roughness because of the wheels' dimensions and the surface of the roughness. So the inconvenience caused should be sought for other reasons. Although some researchers have suspected and 
reported ${ }^{[24,25]}$ the effect of tire bounce, the study in this area did not continue further.

In this paper the problem is studied by another point of view. A possible reason could be the forces developing during the rolling of the wheels on the abnormal surface of the deck-road.

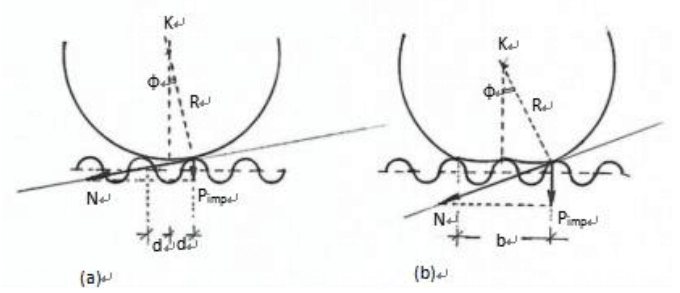

Figure 6. Wheel on roughness

The roughness is considered as a series of repeated small irregularities, which the wheel passes without changing its level of motion but with the development of impact forces from each small irregularity (Figure 6).

It can easily be found that:

$P_{\text {imp }}=\frac{\varepsilon m_{o} v}{2} \cdot \sin \phi=\frac{\varepsilon m_{o} v}{2} \cdot \frac{d}{R}$

with d, determined according to ISO 8608 and 4287 .

Figure $6 \mathrm{a}$ assumes that the wheel will be undeformed, and Figure $6 \mathrm{~b}$ applies when $b>3 d$. In the first case it will be $\tan \phi=d / R$ while in the second one $\tan \phi=b /$ $2 R$.

Assuming that two successive elevations of roughness are $2 \mathrm{~d}$ apart, then when the load enters the $\mathrm{i}^{\text {th }}$ elevation, the mathematical formulation will be:

$G=P_{\text {imp }} \cdot \delta\left(x-a_{i}\right) \cdot \delta\left(t-t_{a_{i}}\right)$

where $\alpha_{i}$ is the distance of the $\mathrm{i}^{\text {th }}$ elevation from the left end of the bridge.

Therefore the corresponding equations of bridge's motion are:

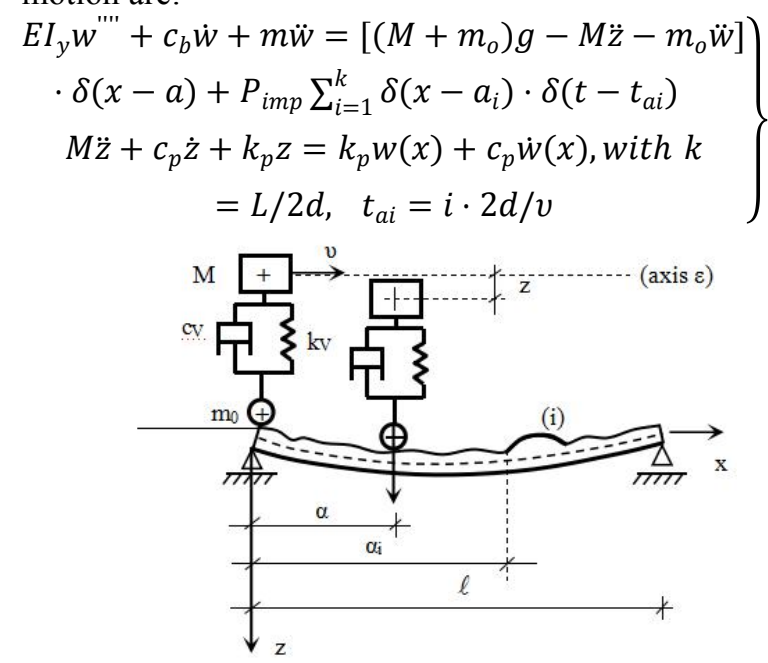

Figure 7. Bridge under the action of a wheel load
Solving the second of equations (15) and following the procedure of $\$ 3.1$, we conclude to the following expressions:

$$
\ddot{z}(t)=\Phi(t)+\frac{1}{\bar{\omega}_{p}} \int_{0}^{t} \Xi(\tau) \cdot \Phi(\tau) d \tau
$$

where: $\Phi(t)=\omega_{p}^{2} w(a, t)+2 \beta_{p} \dot{w}(a, t)$

$$
\left.\begin{array}{c}
\Xi(t)=e^{-\beta_{p}(t-\tau)}\left[\left(\beta_{p}^{2}-\bar{\omega}_{p}^{2}\right) \sin \omega_{p}(t-\tau)\right. \\
+2 \beta_{p} \bar{\omega}_{p} \cos \omega_{p}(t-\tau) \\
\beta_{p}=c_{p} / 2 M, \omega_{p}^{2}=k_{p} / M, \bar{\omega}_{p}=\sqrt{\omega_{p}^{2}-\beta_{p}^{2}}
\end{array}\right\}
$$

In order to solve the first of equations (15), we are searching for a solution of the form:

$w(x, t)=\sum_{n} X_{n}(x) \cdot T_{n}(t)$

where $X_{n}$ are the shape functions of the beam and $T_{n}$ are the time functions to be determined. Introducing $w(x, t)$ from (16b) into the first of equations (15) and taking into account equation (13), we obtain the following equation:

$$
\begin{gathered}
E I_{y} \sum_{n} X_{n}^{\prime \prime \prime} T_{n}+c_{b} \sum_{n} X_{n} \dot{T}_{n}+m \sum_{n} X_{n} \ddot{T}_{n} \\
=\left[\left(M+m_{o}\right) g-M \ddot{z}-m_{o} \sum_{n} X_{n} \ddot{T}_{n}\right] \delta(x-a) \\
+\frac{\varepsilon m_{o} v d}{2 R} \sum_{i=1}^{k} \delta(x-a) \delta\left(t-t_{a}\right) \\
\text { with } \ddot{z} \text { from }(16 a) \text { and } \Phi(t) \\
=\sum_{n} \sin \frac{n \pi x}{L}\left(\omega_{p}^{2} T_{n}+2 \beta_{p} \dot{T}_{n}\right)
\end{gathered}
$$

Because of equations governing the beam's free vibration (as they are exposed in \$3.1), the first of equations $(16 \mathrm{c})$ becomes:

$$
\left.\begin{array}{c}
m \sum_{n} X_{n} \ddot{T}_{n}+c_{b} \sum_{n} X_{n} \dot{T}_{n}+m \sum_{n} \omega_{n}^{2} X_{n} T_{n} \\
=\left[\left(M+m_{o}\right) g-M \ddot{z}-m_{o} \sum_{n} X_{n} \ddot{T}_{n}\right] \delta(x-a) \\
+\frac{\varepsilon m_{o} v d}{2 R} \sum_{i=1}^{k} \delta(x-a) \delta\left(t-t_{a}\right)
\end{array}\right\}
$$

where $\mathrm{k}$ and $t_{a}$ are calculated from equations (15).

Multiplying equation (16d) by $X_{k}(k \neq n)$ and integrating the resultant from 0 to $\mathrm{L}$, we obtain the following equation:

$$
\left.\begin{array}{c}
\ddot{T}_{k}+\frac{c_{b}}{m} \dot{T}_{k}+\omega_{k}^{2} T_{k}=\frac{2}{m L}\left[\left(M+m_{o}\right) g-M \ddot{z}-m_{o}\right. \\
\left.\sum_{n} X_{n} \ddot{T}_{n}\right] \sin \Omega_{k} t \\
+\frac{\varepsilon m_{o} v d}{m L R} \sum_{i=1}^{k} \sin \frac{k \pi \alpha}{L} \delta\left(t-t_{a}\right)
\end{array}\right\}
$$

Using as first approximation the expression of equation (11e), we finally find:

$$
\left.\begin{array}{c}
T_{k}(t)=\frac{2}{m L \bar{\omega}_{k}} \int_{0}^{t}\left[\left(M+m_{o}\right) g-M \ddot{z}(\tau)-m_{o} \sum_{n} \sin \Omega_{n}\right. \\
\left.\tau \overline{\bar{T}}_{n}(\tau)\right] \sin \Omega_{k} \tau \cdot e^{-\beta(t-\tau)} \sin \omega_{k}(t-\tau) d \tau \\
+\frac{\varepsilon m_{o} v d}{m L R \bar{\omega}_{k}} \sum_{i=1}^{k} \sin \Omega_{k} t_{a} \cdot e^{-\beta\left(t-t_{a}\right)} \sin \omega_{k}\left(t-t_{a}\right) H\left(t-t_{a}\right)
\end{array}\right\}
$$

After the impact of the wheel on the $i^{\text {th }}$ irregularity of the roughness, its influence lasts until its impact on the $(\mathrm{i}+1)^{\text {th }}$ one. Then at $t_{i+1}=t_{i}+2 d / v$, the beam vibrates under the action of $P_{i m p}$ on $(i+1)^{\text {th }}$ irregularity and under the appeared free motion due to the previous action of 
$P_{i m p}$ on the $\mathrm{i}^{\text {th }}$ irregularity. This last term for the $\mathrm{k}$ mode has the following time function:

$T_{k}^{f r e e}(t)=\frac{2 P_{i m p}}{m L \omega_{k}} \cdot e^{-\beta\left(t-t_{i}\right)}\left[A_{k} \sin \omega_{k}\left(t-t_{i}\right)+B_{k} \cos \omega_{k}(t-\right.$ $\left.\left.t_{i}\right)\right]$

Using the time conditions

$T_{k}\left(t_{i}\right)=T_{k}^{\text {free }}\left(t_{i}\right)$, and $\left.\dot{T}_{k}\left(t_{i}\right)=\dot{T}_{k}^{\text {free }}\left(t_{i}\right)\right\}$

We finally find:

$\left.A_{k}=e^{-\beta\left(t_{i}-t_{i-1}\right)} \cdot \sin \Omega_{k} t_{i-1} \cdot \cos \omega_{k}\left(t_{i}-t_{i-1}\right)\right\}$

$\left.B_{k}=e^{-\beta\left(t_{i}-t_{i-1}\right)} \cdot \sin \Omega_{k} t_{i-1} \cdot \sin \omega_{k}\left(t_{i}-t_{i-1}\right)\right\}$

\section{Damped Vehicle}

\subsection{Irregularities}

Let us now consider the vehicle of Figure 3c.

Assuming that the weight of the vehicle is equally distributed between the front and back wheels, the forces applied on the bridge will be:

$$
\left.\begin{array}{c}
F_{1}=\left[\frac{M}{2}(g-\ddot{z})+m_{o}(g-\ddot{w})\right] \delta(x-a) \\
+\frac{\varepsilon m_{o} v}{2} \sin \phi \sum_{i=1}^{k} \delta(x-a) \delta\left(t-t_{a}\right) \\
F_{2}=\left[\frac{M}{2}(g-\ddot{z})+m_{o}(g-\ddot{w})\right] \delta\left(x-a+D_{e}\right) \\
+\frac{\varepsilon m_{o} v}{2} \sin \phi \sum_{i=1}^{k} \delta\left(x-a+D_{e}\right) \delta\left(t-t_{a}\right)
\end{array}\right\}
$$

where $\ddot{Z}$ derives from equation (11d), and $D_{e}$ the wheelbase of the vehicle.

Searching for a solution under the form: $w(x, t)=\sum_{n} X_{n}(x) \cdot T_{n}(t)$

using the approximate solution of equation (11e) and following the known procedure, we determine the time function $T_{k}(t)$ as follows:

$$
\begin{aligned}
& T_{k}=\frac{2}{m L \bar{\omega}_{k}} \int_{0}^{t} G_{1}(\tau) e^{-\beta(t-\tau)} \sin \omega_{k}(t-\tau) d \tau+\frac{\varepsilon m_{o} v}{2 m \bar{\omega}_{k}} \\
& \sin \phi \sum_{i=1}^{k} \sin \Omega_{k} t_{a 1} e^{-\beta\left(t-t_{a 1}\right)} \sin \omega_{k}\left(t-t_{a 1}\right) H\left(t-t_{a 1}\right) \\
& +\frac{2}{m L \bar{\omega}_{k}} \int_{0}^{t} G_{2}(\tau) e^{-\beta(t-\tau)} \sin \omega_{k}(t-\tau) d \tau+\frac{\varepsilon m_{o} v}{2 m \bar{\omega}_{k}} \\
& \left.\sin \phi \sum_{i=1}^{k} \sin \Omega_{k} t_{a 2} e^{-\beta\left(t-t_{a 2}\right)} \sin \omega_{k}\left(t-t_{a 2}\right) H\left(t-t_{a 2}\right)\right) \\
& \text { where: } \\
& G_{1}(t)=\left[\left(\frac{M}{2}+m_{o}\right) g-\frac{M}{2} \ddot{z}(t)-m_{o} \sum_{n} \sin \Omega_{n} t_{1} \ddot{\bar{T}}_{n}(t)\right] \\
& \sin \Omega_{k} t_{1} \\
& G_{2}(t)=\left[\left(\frac{M}{2}+m_{o}\right) g-\frac{M}{2} \ddot{z}(t)-m_{o} \sum_{n} \sin \Omega_{n} t_{2} \ddot{\bar{T}}_{n}(t)\right] \\
& \sin \Omega_{k} t_{2} \\
& t_{a 1}=\frac{\delta_{i}+\xi_{o}}{v}, t_{a 2}=\frac{\delta_{i}+\xi_{o}-D_{e}}{v}, t_{1}=\frac{\delta_{i}}{v}, t_{2}=\frac{\delta_{i}-D_{e}}{v}
\end{aligned}
$$

\subsection{Roughness}

Under the assumptions of $\S 4.1$, we have the following loads applied on the bridge:

$$
\left.\begin{array}{c}
F_{1}=\left[\frac{M}{2}(g-\ddot{z})+m_{o}(g-\ddot{w})\right] \delta(x-a)+\frac{\varepsilon m_{o} v d}{2 R} \\
\sum_{i=1}^{k} \delta(x-a) \delta\left(t-t_{a 1}\right) \\
F_{2}=\left[\frac{M}{2}(g-\ddot{z})+m_{o}(g-\ddot{w})\right] \delta\left(x-a+D_{e}\right)+\frac{\varepsilon m_{o} v d}{2 R} \\
\sum_{i=1}^{k} \delta\left(x-a+D_{e}\right) \delta\left(t-t_{a 2}\right)
\end{array}\right\}
$$

We are looking again for a solution under the form: $w(x, t)=\sum_{n} X_{n}(x) \cdot T_{n}(t)$

Using the approximate solution of equation (11e) and following the known procedure, we determine the time function $T_{k}(t)$ as follows:

$$
\left.\begin{array}{c}
T_{k}=\frac{2}{m L \bar{\omega}_{k}} \int_{0}^{t} G_{1}(\tau) e^{-\beta(t-\tau)} \sin \omega_{k}(t-\tau) d \tau+\frac{\varepsilon m_{o} v d}{2 R m \bar{\omega}_{k}} \sin \phi \\
\sum_{i=1}^{k} \sin \Omega_{k} t_{1} e^{-\beta\left(t-t_{1}\right)} \sin \omega_{k}\left(t-t_{1}\right) H\left(t-t_{1}\right) \\
+\frac{2}{m L \bar{\omega}_{k}} \int_{0}^{t} G_{2}(\tau) e^{-\beta(t-\tau)} \sin \omega_{k}(t-\tau) d \tau+\frac{\varepsilon m_{o} v d}{2 R m \bar{\omega}_{k}} \sin \phi \\
\sum_{i=1}^{k} \sin \Omega_{k} t_{2} e^{-\beta\left(t-t_{2}\right)} \sin \omega_{k}\left(t-t_{2}\right) H\left(t-t_{2}\right)
\end{array}\right\}
$$

where $\ddot{z}$ and $\Phi(t)$ are calculated from equation (16a), and $G_{1}(t), G_{2}(t)$ from (18d).

Finally:

$\left.t_{1}=i \cdot \frac{2 d}{v}, \quad t_{2}=i \cdot \frac{2 d}{v}-D_{e}\right\}$

\section{Numerical Results and Discussion}

The purpose of this paper is to study the influence of some parameters on the dynamic response of a bridge through a new approach in particular for the case of roughness. The disorder caused by roughness is taken into account as a result of the appearance of impact forces.

The paper focuses on the following parameters:

a. The length and the position of a set of consecutive or distant irregularities.

b. The quality of the deck surface, which means the size of the roughness gaps and the caused trouble to the passengers of the car.

Therefore we have studied two types of bridges and vehicles, which are described below. Note should be taken of the following:

- The vehicles are supposed to move along the center line of the bridge. Thus no rotational motion is developed.

- The displacements of the bridge in the middle of the span are studied.

- Only the first six flexural modes are taken into account.

\subsection{Data}

\subsubsection{Bridges}

The bridges are made from homogeneous and isotropic material, having modulus of elasticity $E=2.1 \cdot 10^{10} \mathrm{dN} /$ $m^{2}$.

Let us consider two kinds of bridges.

- The one, relatively short bridge of length $L=20 \mathrm{~m}$, mass per unit length $m=250 \mathrm{~kg} / \mathrm{m}$, and moment of inertia $I_{y}=0.01 \mathrm{~m}^{4}$

- and the other, relatively long bridge of length $L=$ $80 \mathrm{~m}$, mass per unit length $m=350 \mathrm{~kg} / \mathrm{m}$, and moment of inertia $I_{y}=0.80 \mathrm{~m}^{4}$. 


\subsubsection{Vehicles}

The passage of two types of vehicle is studied. The constants of the spring $k_{0}$ are taken from the Kraemer and Freund catalogues and those of the dampers $c_{o}$ from the Sachs-Stabilus ones.

The characteristics of the above two types of vehicles are:

$$
\begin{array}{ll}
\underline{\text { Light Vehicle }} & \text { Big Lorry } \\
M=300 \mathrm{~kg} & M=2500 \mathrm{~kg} \\
m_{o}=8 \mathrm{~kg} & m_{o}=20 \mathrm{~kg} \\
m_{o}=8 \mathrm{~kg} & m_{o}=20 \mathrm{~kg} \\
R=0.30 \mathrm{~m} & R=0.40 \mathrm{~m} \\
k_{o}=6000 \mathrm{dN} / \mathrm{m} & k_{o}=10000 \mathrm{dN} / \mathrm{m} \\
c_{o}=100 \mathrm{dN} \cdot \mathrm{sec} / \mathrm{m} & c_{o}=500 \mathrm{dN} \cdot \mathrm{sec} / \mathrm{m}
\end{array}
$$

\subsubsection{Irregularities and Roughness}

Two types of irregularities, in relation to their shape, were taken into consideration. Both have the same length $b_{i}=$ $1.00 \mathrm{~m}$, while their height is 0.05 and $0.20 \mathrm{~m}$ respectively. Their place is on the right end or in the middle of the span.

As for the roughness study three kinds of pavement are considered. The soft one with $d=0.01 \mathrm{~m}$, the middle one with $d=0.02 \mathrm{~m}$, and the bad one with $d=0.035 \mathrm{~m}$. Lastly the case of a cobbled road is studied with $d=$ $0.10 m$.

\subsection{Irregularities}

Applying the formulae of paragraphs 3.1 and 4.1, we obtain the following diagrams related to the motion of the middle of the bridge, for different irregularities (form and location), length of the bridge and kind of model (vehicle).

\subsubsection{Short Bridge - Light Vehicle}

In the following Figure 8 we see the plots showing the motion of the middle of a short bridge due to the passage of various models of moving loads with speed $20 \mathrm{~m} / \mathrm{sec}$ and under the following conditions: successive high irregularities $(\beta=1.00 \mathrm{~m}, h=0.20 \mathrm{~m})$, located at the left end of the bridge with $\alpha_{1}=1 \mathrm{~m}, a_{2}=3 \mathrm{~m}$.

As it was expected, the use of a relatively more accurate model shows differences that can be amounted to more than $15 \%$.

In the following Figure 9 we see the plots showing the motion of the middle of a short bridge due to the passage of various models of moving loads with speed $20 \mathrm{~m} / \mathrm{sec}$ and under the following conditions: successive high irregularities $(\beta=1.00 m, h=0.20 m)$, located in the middle of the bridge with $\alpha_{1}=11 \mathrm{~m}, a_{2}=13 \mathrm{~m}$.
As it was expected, the use of a relatively more accurate model shows differences that can be amounted to more than $10 \%$.

In the following Figure 10 we see the plots showing the motion of the middle of a short bridge due to the passage of various models of moving loads with speed 20 $\mathrm{m} / \mathrm{sec}$ and under the following conditions: successive low irregularities $(\beta=1.00 \mathrm{~m}, h=0.05 \mathrm{~m})$, located at the left end of the bridge with $\alpha_{1}=1 m, a_{2}=3 m$.

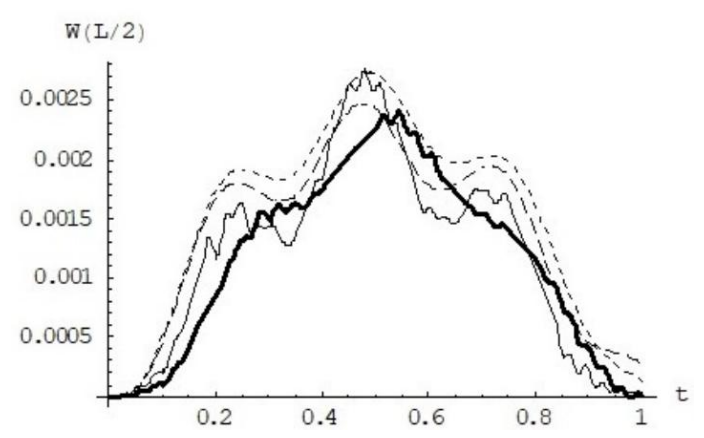

Figure 8. (high irregularity), w(in $\mathrm{m}), \mathrm{t}$ (in sec) ....... MODEL 1. Bridge without irregularities, - - - - MODEL 2. Bridge without irregularities - MODEL 2. Bridge with irregularities,
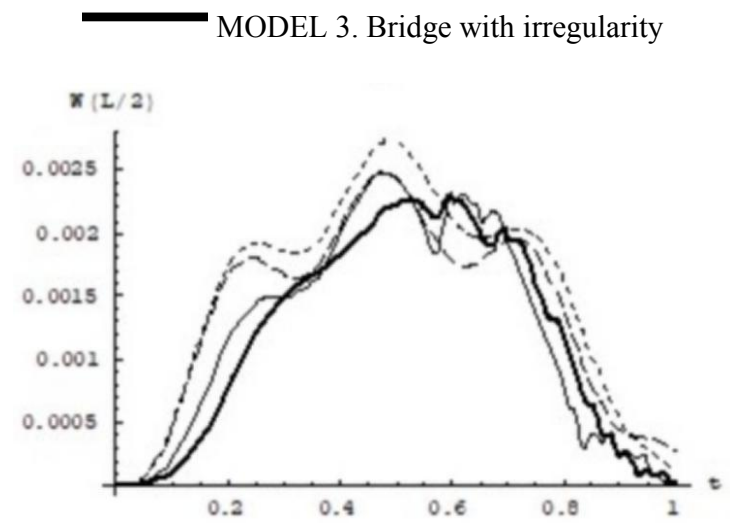

Figure 9. (high irregularity), w(in $\mathrm{m}), \mathrm{t}$ (in sec) ....... MODEL 1. Bridge without irregularities, - - - MODEL 2. Bridge without irregularities - MODEL 2. Bridge with irregularities, MODEL 3. Bridge with irregularity

We verify also the necessity to use more accurate models.

In the following Figure 11 we see the plots showing the motion of the middle of a short bridge due to the passage of various models of moving loads with speed 20 $\mathrm{m} / \mathrm{sec}$ and under the following conditions: successive low irregularities $(\beta=1.00 \mathrm{~m}, h=0.05 \mathrm{~m})$, located in the middle of the bridge with $\alpha_{1}=11 \mathrm{~m}, a_{2}=13 \mathrm{~m}$. 


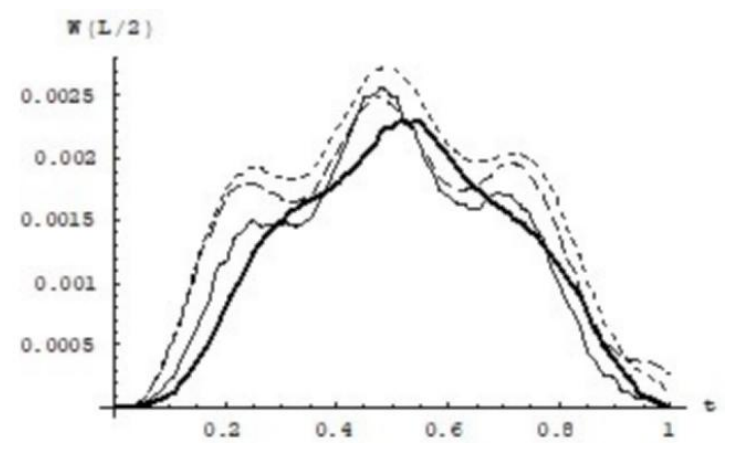

Figure 10. (low irregularity), w(in $\mathrm{m})$, $\mathrm{t}($ in $\mathrm{sec})$

...... MODEL 1. Bridge without irregularities,

- - - MODEL 2. Bridge without irregularities MODEL 2. Bridge with irregularities,

MODEL 3. Bridge with irregularity

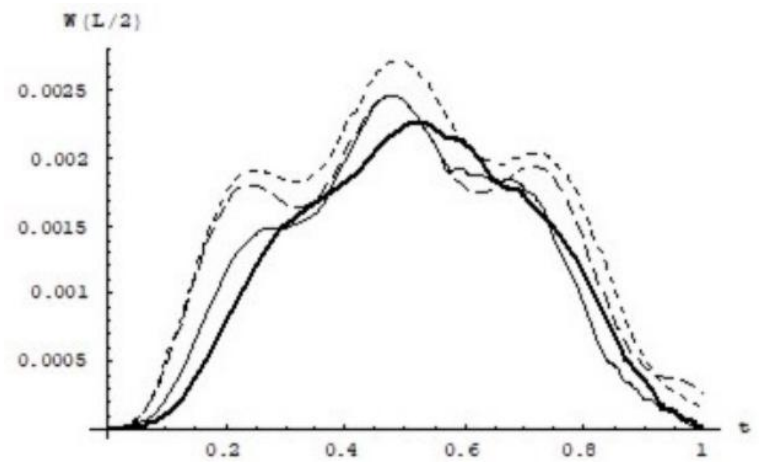

Figure 11. (low irregularity), w(in $\mathrm{m}), \mathrm{t}$ (in sec)

...... MODEL 1. Bridge without irregularities,

- - - MODEL 2. Bridge without irregularities

- MODEL 2. Bridge with irregularities,

MODEL 3. Bridge with irregularity

\subsubsection{Short Bridge - Big Lorry}

In the following Figure 12 we see the plots showing the motion of the middle of a short bridge due to the passage of various models of moving loads with speed 15 $\mathrm{m} / \mathrm{sec}$ and under the following conditions: successive high irregularities $(\beta=1.00 \mathrm{~m}, h=0.20 \mathrm{~m})$, located at the left end of the bridge with $\alpha_{1}=1 \mathrm{~m}, a_{2}=3 \mathrm{~m}$.

As it was expected, the use of a relatively more accurate model shows differences that can be amounted to more than $25 \%$ for higher speeds.

In the following Figure 13 we see the plots showing the motion of the middle of a short bridge due to the passage of various models of moving loads with speed 15 $\mathrm{m} / \mathrm{sec}$ and under the following conditions: successive high irregularities $(\beta=1.00 \mathrm{~m}, h=0.20 \mathrm{~m})$, located in the middle of the bridge with $\alpha_{1}=11 \mathrm{~m}, a_{2}=13 \mathrm{~m}$.

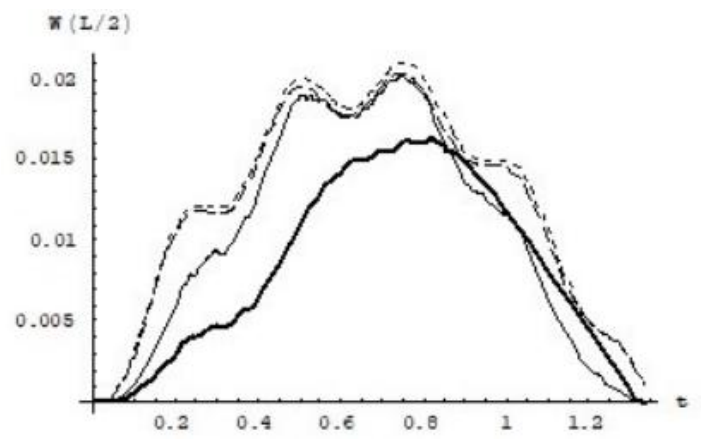

Figure12. (high irregularity), w(in $\mathrm{m}), \mathrm{t}($ in $\mathrm{sec})$ ...... MODEL 1. Bridge without irregularities, - - - MODEL 2. Bridge without irregularities - MODEL 2. Bridge with irregularities,
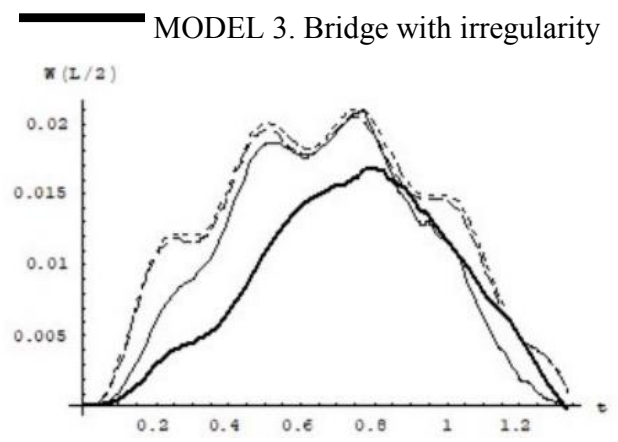

Figure 13. (high irregularity), w(in $\mathrm{m}), \mathrm{t}($ in sec) ...... MODEL 1. Bridge without irregularities, - - - MODEL 2. Bridge without irregularities - MODEL 2. Bridge with irregularities, MODEL 3. Bridge with irregularity

The use of accurate models seems to be also necessary. In the following Figure 14 we see the plots showing the motion of the middle of a short bridge due to the passage of various models of moving loads with speed 15 $\mathrm{m} / \mathrm{sec}$ and under the following conditions: successive low irregularities $(\beta=1.00 \mathrm{~m}, h=0.05 \mathrm{~m})$, located at the left end of the bridge with $\alpha_{1}=1 \mathrm{~m}, a_{2}=3 \mathrm{~m}$.

In the following Figure 15 we see the plots showing the motion of the middle of a short bridge due to the passage of various models of moving loads with speed 15 $\mathrm{m} / \mathrm{sec}$ and under the following conditions: successive low irregularities $(\beta=1.00 \mathrm{~m}, h=0.05 \mathrm{~m})$, located in the middle of the bridge with $\alpha_{1}=11 \mathrm{~m}, a_{2}=13 \mathrm{~m}$.

\subsubsection{Long Bridge - Light Vehicle}

In the following Figure 16 we see the plots showing the motion of the middle of a long bridge due to the passage of various models of moving loads with speed 20 $\mathrm{m} / \mathrm{sec}$ and under the following conditions: successive high 
irregularities $(\beta=1.00 \mathrm{~m}, h=0.20 \mathrm{~m})$, located at the left end of the bridge with $\alpha_{1}=1 \mathrm{~m}, a_{2}=3 \mathrm{~m}$.

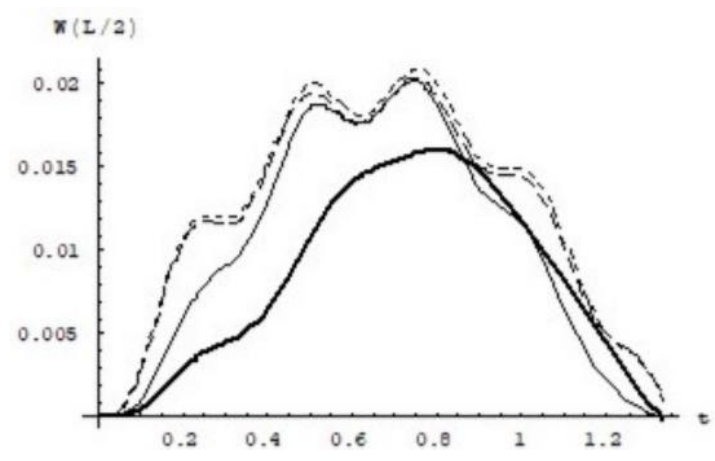

Figure 14. (low irregularity), $w($ in $m), t($ in sec)

...... MODEL 1. Bridge without irregularities,

- - - - MODEL 2. Bridge without irregularities

- MODEL 2. Bridge with irregularities,

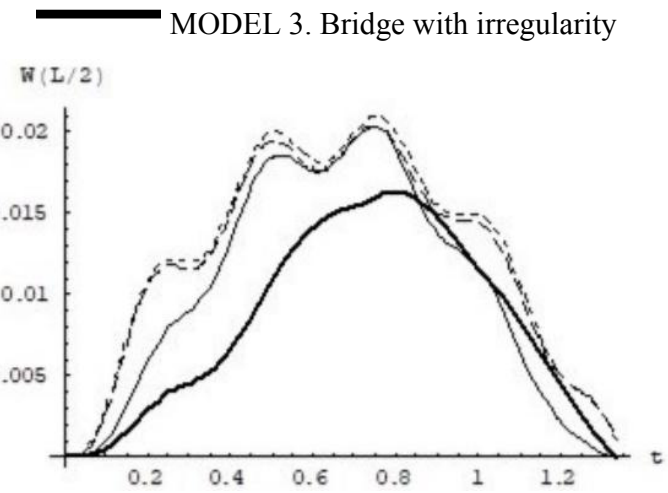

Figure 15. (low irregularity), w(in $\mathrm{m})$, $\mathrm{t}($ in sec)

...... MODEL 1. Bridge without irregularities,

- - - - MODEL 2. Bridge without irregularities

MODEL 2. Bridge with irregularities,

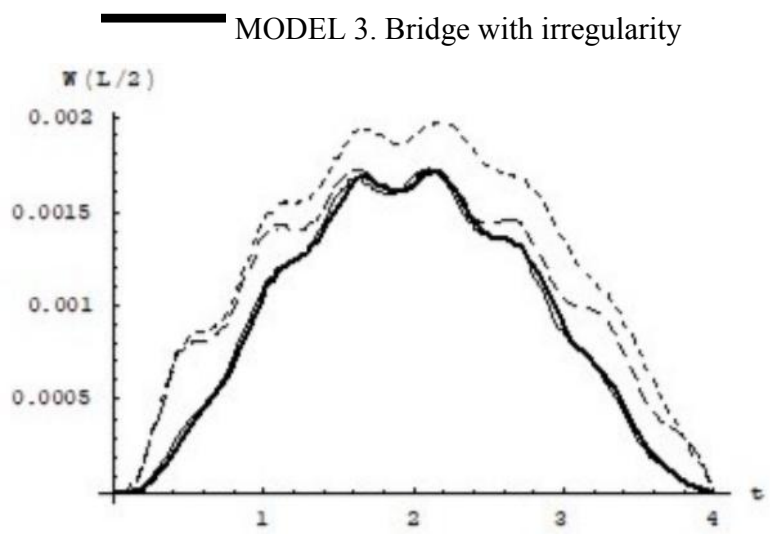

Figure 16. (high irregularity), w(in $\mathrm{m}$ ), $\mathrm{t}($ in $\mathrm{sec}$ ) MODEL 1. Bridge without irregularities,

- - - MODEL 2. Bridge without irregularities

- MODEL 2. Bridge with irregularities,

MODEL 3. Bridge with irregularity
In the following Figure 17 we see the plots showing the motion of the middle of a long bridge due to the passage of various models of moving loads with speed 20 $\mathrm{m} / \mathrm{sec}$ and under the following conditions: successive high irregularities $(\beta=1.00 \mathrm{~m}, h=0.20 \mathrm{~m})$, located in the middle of the bridge with $\alpha_{1}=38 \mathrm{~m}, a_{2}=41 \mathrm{~m}$.

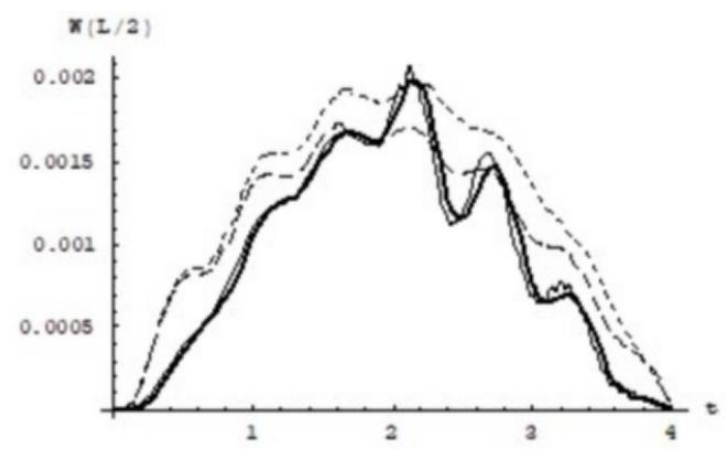

Figure 17. (high irregularity), w(in $\mathrm{m}$ ), $\mathrm{t}$ (in sec)

....... MODEL 1. Bridge without irregularities,

- - - - MODEL 2. Bridge without irregularities

- MODEL 2. Bridge with irregularities,

MODEL 3. Bridge with irregularity

In the following Figure 18 we see the plots showing the motion of the middle of a long bridge due to the passage of various models of moving loads with speed 20 $\mathrm{m} / \mathrm{sec}$ and under the following conditions: successive low irregularities $(\beta=1.00 \mathrm{~m}, h=0.05 \mathrm{~m})$, located at the left end of the bridge with $\alpha_{1}=1 \mathrm{~m}, a_{2}=3 \mathrm{~m}$.

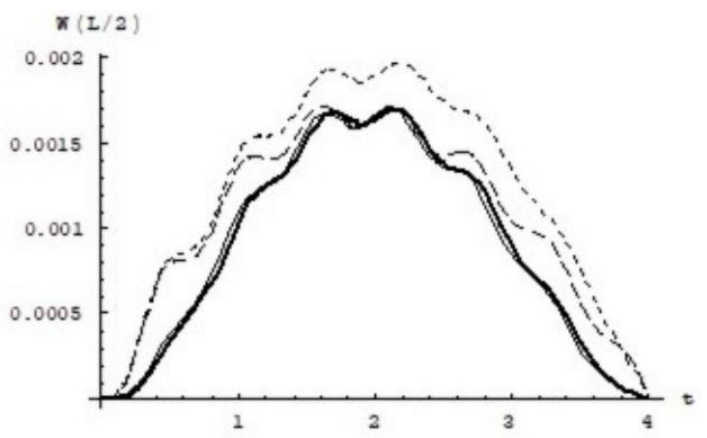

Figure18. (low irregularity), w(in $\mathrm{m}), \mathrm{t}$ (in sec)

...... MODEL 1. Bridge without irregularities,

- - - MODEL 2. Bridge without irregularities

- MODEL 2. Bridge with irregularities,

MODEL 3. Bridge with irregularity

In the following Figure 19 we see the plots showing the motion of the middle of a long bridge due to the passage of various models of moving loads with speed 20 $\mathrm{m} / \mathrm{sec}$ and under the following conditions: successive low irregularities $(\beta=1.00 \mathrm{~m}, h=0.05 \mathrm{~m})$, located in the middle of the bridge with $\alpha_{1}=38 \mathrm{~m}, a_{2}=41 \mathrm{~m}$. 


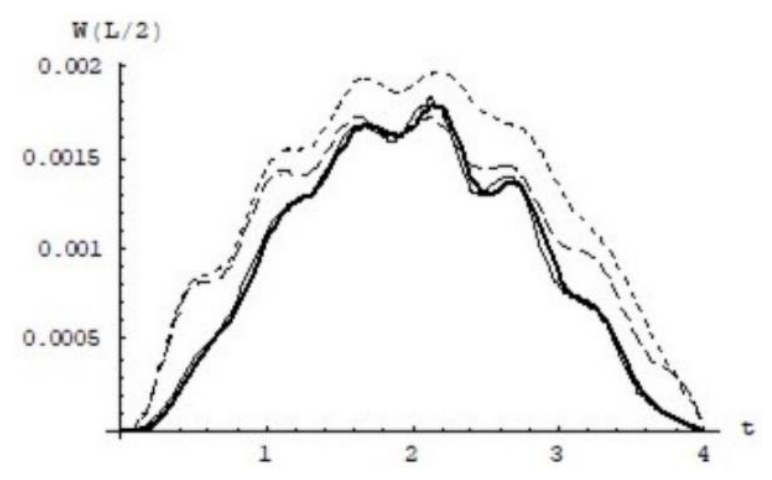

Figure 19. (low irregularity), w(in $\mathrm{m}), \mathrm{t}($ in $\mathrm{sec})$

...... MODEL 1. Bridge without irregularities,

- - - MODEL 2. Bridge without irregularities

MODEL 2. Bridge with irregularities,

MODEL 3. Bridge with irregularity

\subsubsection{Long Bridge - Big Lorry}

In the following Figure 20 we see the plots showing the motion of the middle of a long bridge due to the passage of various models of moving loads with speed 15 $\mathrm{m} / \mathrm{sec}$ and under the following conditions: successive high irregularities $(\beta=1.00 m, h=0.20 m)$, located at the left end of the bridge with $\alpha_{1}=1 \mathrm{~m}, a_{2}=3 \mathrm{~m}$.

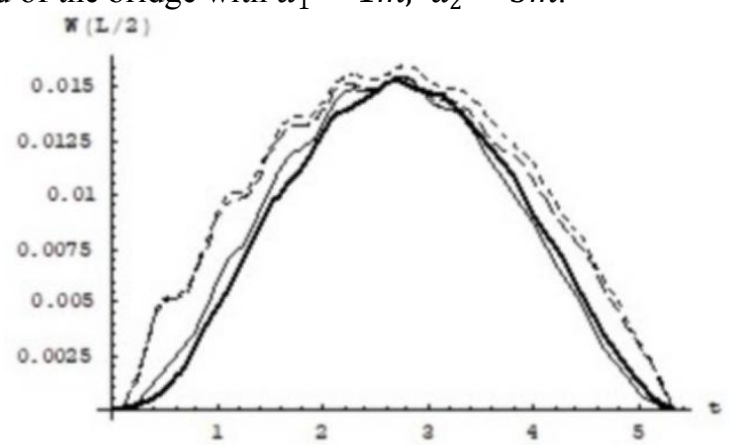

Figure 20. (high irregularity), w(in $\mathrm{m})$, $\mathrm{t}$ (in sec)

...... MODEL 1. Bridge without irregularities,

- - - MODEL 2. Bridge without irregularities

-MODEL 2. Bridge with irregularities,

MODEL 3. Bridge with irregularity

In the following Figure 21 we see the plots showing the motion of the middle of a long bridge due to the passage of various models of moving loads with speed 15 $\mathrm{m} / \mathrm{sec}$ and under the following conditions: successive high irregularities $(\beta=1.00 \mathrm{~m}, h=0.20 \mathrm{~m})$, located in the middle of the bridge with $\alpha_{1}=38 \mathrm{~m}, a_{2}=41 \mathrm{~m}$.

In the following Figure 22 we see the plots showing the motion of the middle of a long bridge due to the passage of various models of moving loads with speed 15 $\mathrm{m} / \mathrm{sec}$ and under the following conditions: successive low irregularities $(\beta=1.00 m, h=0.05 m)$, located at the left end of the bridge with $\alpha_{1}=1 \mathrm{~m}, a_{2}=3 \mathrm{~m}$.

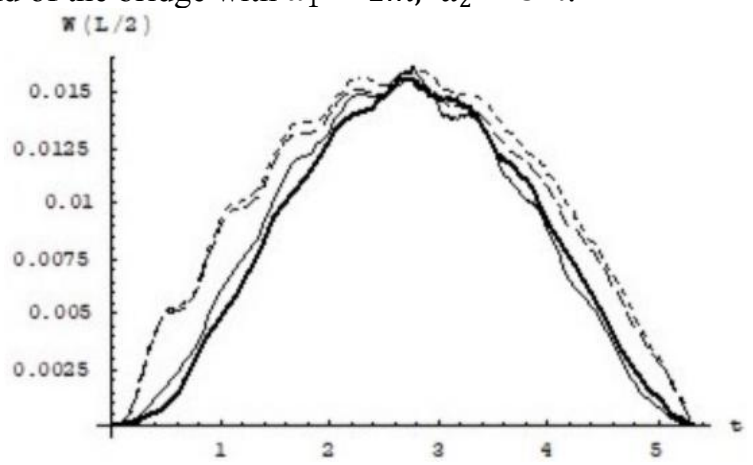

Figure 21. (high irregularity), w(in $\mathrm{m}), \mathrm{t}($ in $\mathrm{sec})$

...... MODEL 1. Bridge without irregularities,

- - - MODEL 2. Bridge without irregularities

- MODEL 2. Bridge with irregularities,

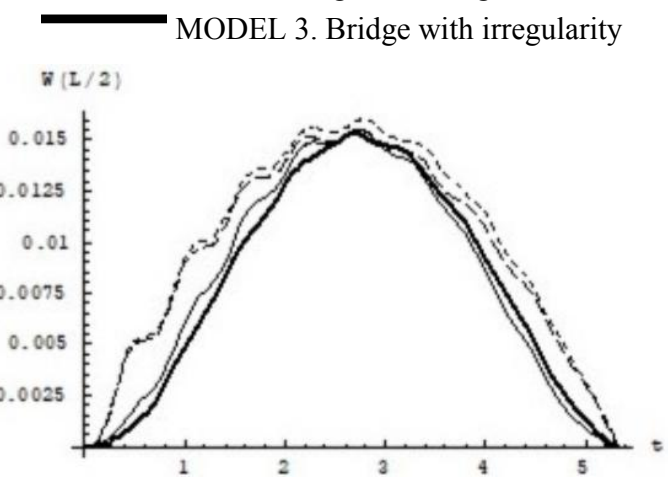

Figure 22. (low irregularity), w(in $\mathrm{m}), \mathrm{t}($ in $\mathrm{sec})$

...... MODEL 1. Bridge without irregularities,

- - - MODEL 2. Bridge without irregularities

- MODEL 2. Bridge with irregularities,

MODEL 3. Bridge with irregularity

In the following Figure 23 we see the plots showing the motion of the middle of a long bridge due to the passage of various models of moving loads with speed 15 $\mathrm{m} / \mathrm{sec}$ and under the following conditions: successive low irregularities $(\beta=1.00 m, h=0.05 m)$, located in the middle of the bridge with $\alpha_{1}=38 \mathrm{~m}, a_{2}=41 \mathrm{~m}$.

We observe that the differences produced by the different models of vehicle are very small ranging from 2 to $5 \%$.

\subsection{Roughness}

Let us consider now three road surface qualities, the practically smooth $(d \leq 0.01 \mathrm{~m})$, the middle $(0.01 \leq$ $d \leq 0.03 m)$ and the bad surface $(d \geq 0.03 m)$.

Applying the formulae of paragraphs 3.2 and 4.2, we obtain the following diagrams related to the motion of the middle of the bridge for a vehicle moving with speed $v=$ 
$10 \mathrm{~m} / \mathrm{sec}$, different kind of roughness, length of the bridge and kind vehicles.

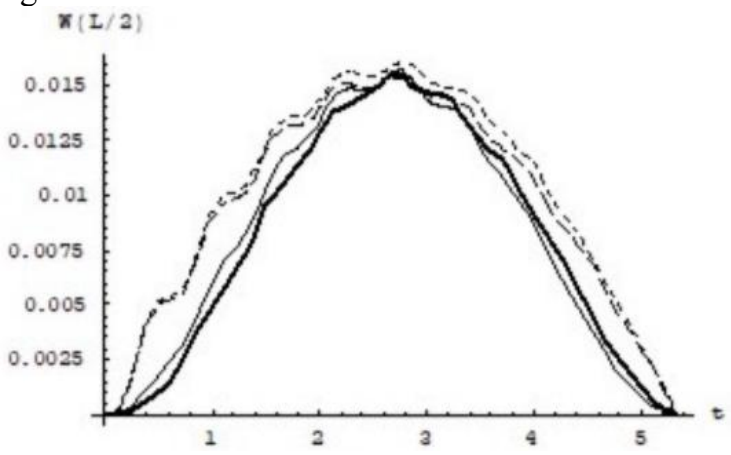

Figure 23. (low irregularity), w(in $\mathrm{m}), \mathrm{t}$ (in sec) ....... MODEL 1. Bridge without irregularities, - - - - MODEL 2. Bridge without irregularities - MODEL 2. Bridge with irregularities, MODEL 3. Bridge with irregularity

\subsubsection{Short Bridge - Light vehicle}

In the following Figure 24 we see the plots showing the motion of the middle of a short bridge due to the passage of a light vehicle moving with speed $10 \mathrm{~m} / \mathrm{sec}$ on a road surface with smooth roughness $(d=0.01 \mathrm{~m})$.

In the following Figure 25 we see the plots showing the motion of the middle of a short bridge due to the passage of a light vehicle moving with speed $10 \mathrm{~m} / \mathrm{sec}$ on a road surface with middle roughness $(d=0.02 m)$.

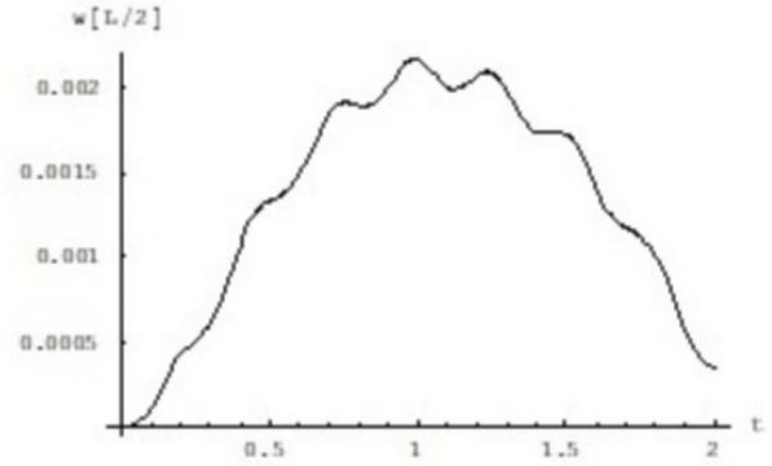

a)

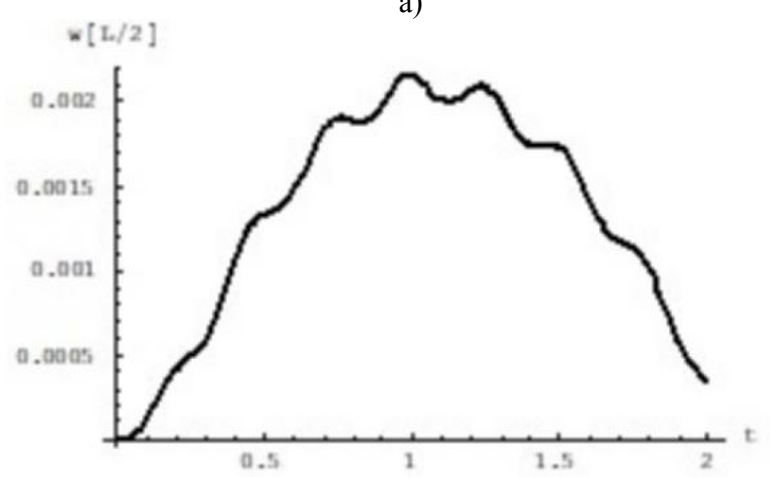

b)

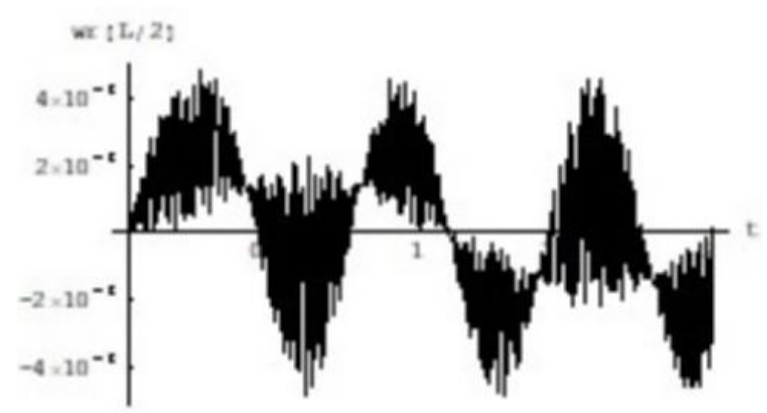

c)

Figure 24. (Roughness 0,01 ), w(in $\mathrm{m}), \mathrm{t}($ in sec) a) Bridge without roughness b) Bridge with roughness c) Deformation of the bridge due to roughness only

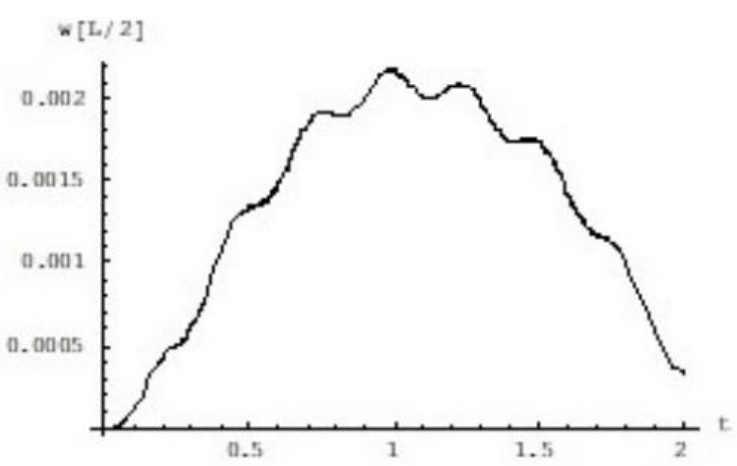

a)

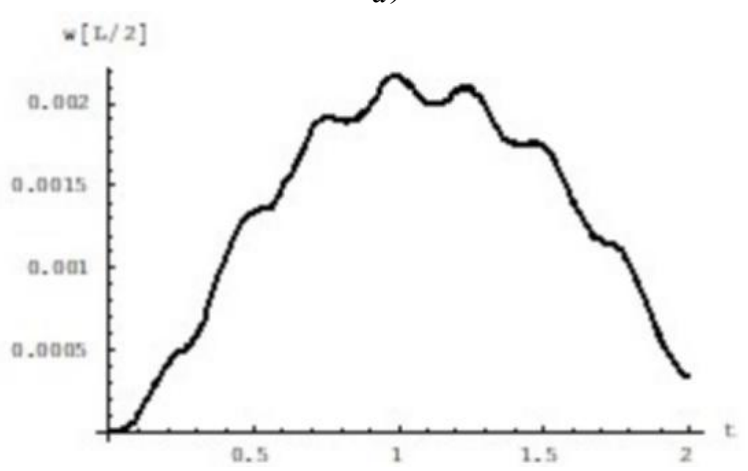

b)

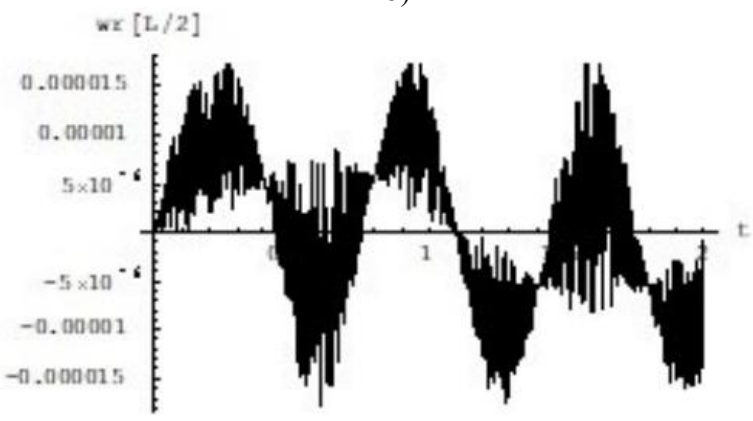

c)

Figure 25. (Roughness 0,02 ), w(in $\mathrm{m}), \mathrm{t}($ in sec) a) Bridge without roughness $b$ ) Bridge with roughness c) Deformation of the bridge due to roughness only 
In the following Figure 26 we see the plots showing the motion of the middle of a short bridge due to the passage of a light vehicle moving with speed $10 \mathrm{~m} / \mathrm{sec}$ on a road surface with bad roughness $(d=0.04 m)$.

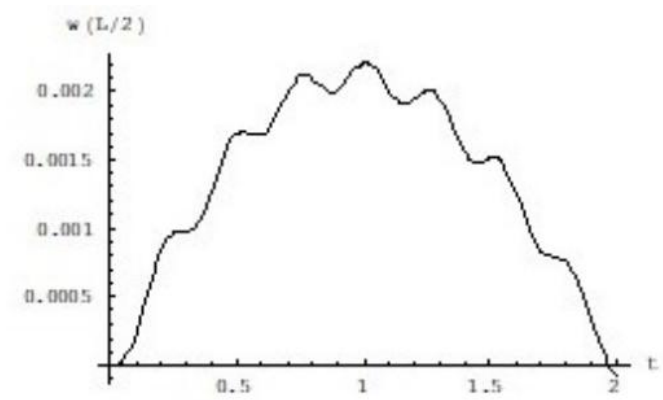

a)

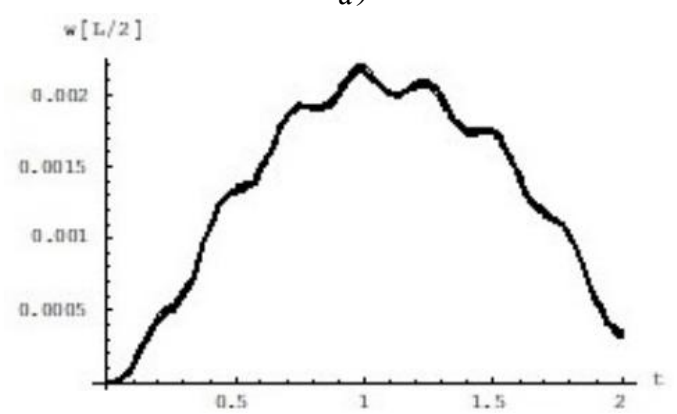

b)

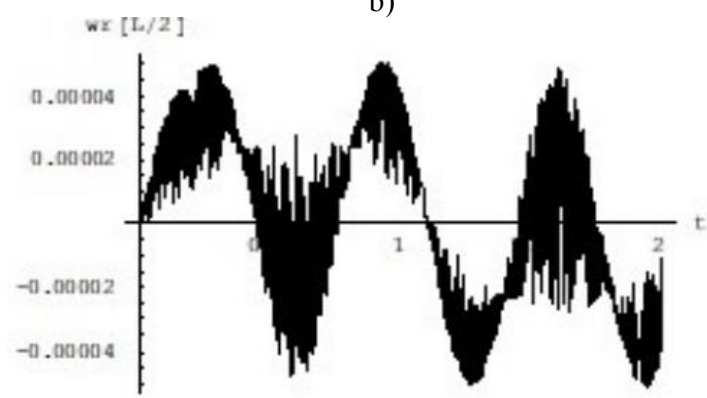

c)

Figure 26. (Roughness 0,04), w(in $\mathrm{m})$, $\mathrm{t}($ in sec)

a) Bridge without roughness b) Bridge with roughness c)

Deformation of the bridge due to roughness only

In the following Figure 27 we see the plots showing the motion of the middle of a short bridge due to the passage of a light vehicle moving with speed $10 \mathrm{~m} / \mathrm{sec}$ on a surface of cobbled road $(d=0.10 \mathrm{~m})$.

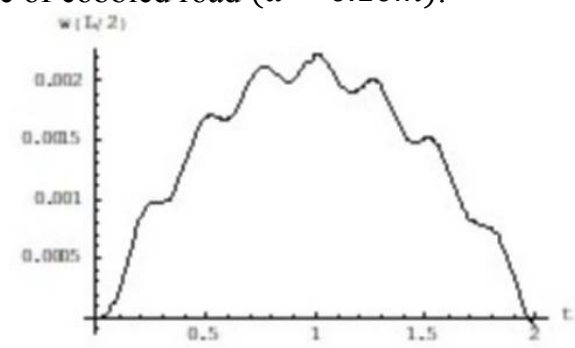

a)

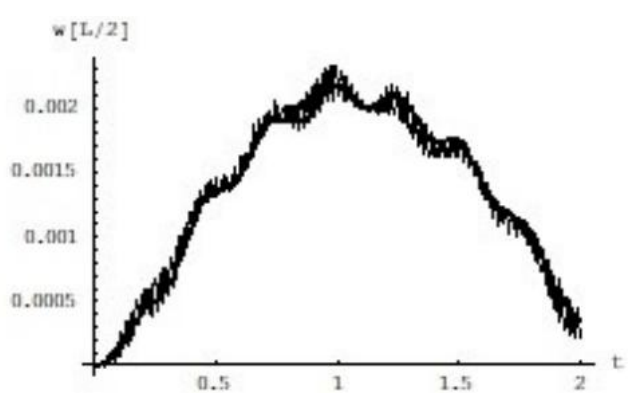

b)

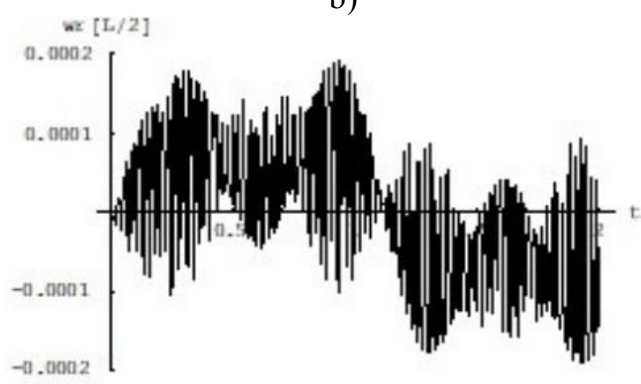

c)

Figure 27. (Roughness 0,10), w(in $\mathrm{m})$, $\mathrm{t}($ in $\mathrm{sec}$ ) a)Bridge without roughness b) Bridge with roughness c) Deformation of the bridge due to roughness only

\subsubsection{Short Bridge - Big Lorry}

In this section we examine the worst case of a road surface with bad roughness $(d=0.04 m)$.

Therefore in the following Figure 28 we see the plots showing the motion of the middle of a short bridge due to the passage of a big lorry moving with speed $10 \mathrm{~m} / \mathrm{sec}$ on a surface with bad roughness.

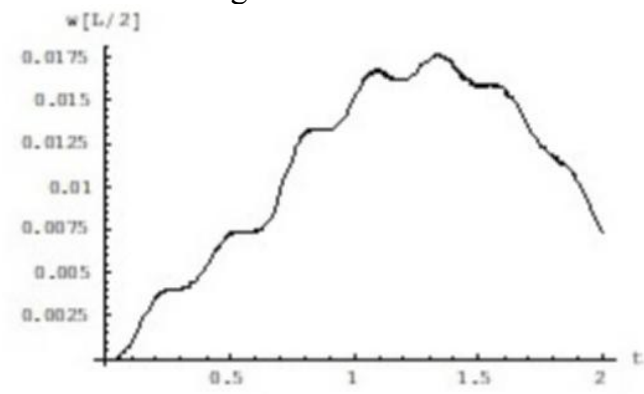

a)

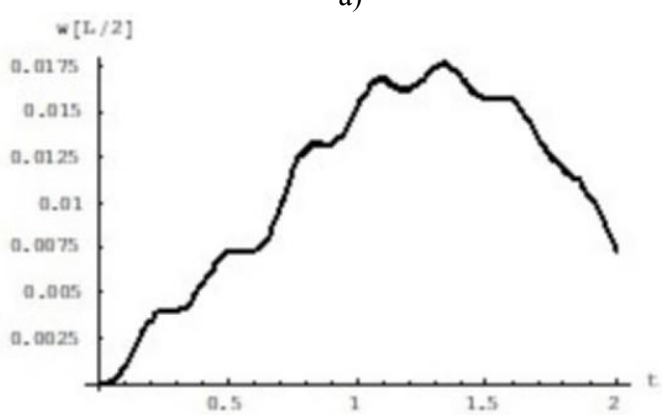

b) 


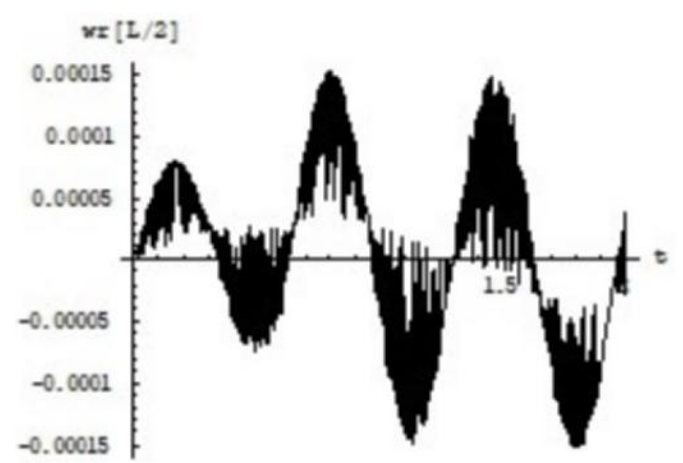

c)

Figure 28. (Roughness 0,04), w(in $\mathrm{m})$, $\mathrm{t}($ in sec)

a) Bridge without roughness b) Bridge with roughness

c) Deformation of the bridge due to roughness only

It is obvious that one can very easily draw analogous plots for the cases of a Long Bridge-short vehicle and a Long Bridge-big lorry, applying the formulae of $\S 3$ and 4.

We believe that it is not necessary to show these plots because the motion of a long bridge due to roughness alone is much smaller than the corresponding motion of a short bridge due to roughness alone.

In the contrary, the following study is interesting.

\subsubsection{Influence of Speed}

We consider that passengers' trouble can be expressed by the breadth of the motion due to roughness alone. Therefore in the plots of Figures 29 and 30 the parts of Figures $24 \mathrm{c}$ to $27 \mathrm{c}$ are presented, showing the maximum motions due to roughness alone, concerning a short and a long bridge respectively.

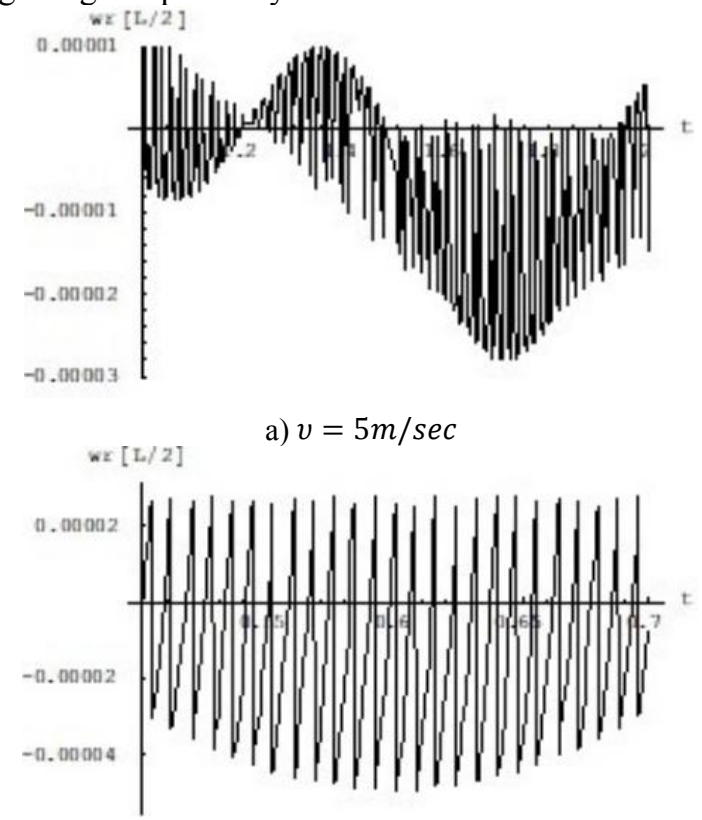

b) $v=10 \mathrm{~m} / \mathrm{sec}$

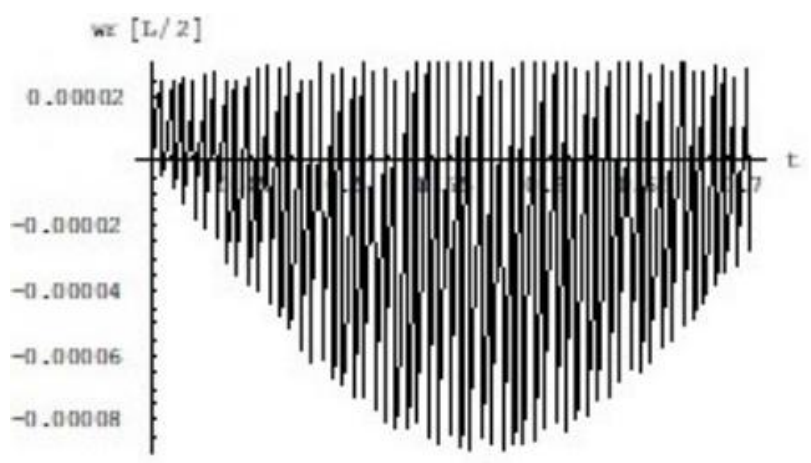

c) $v=15 \mathrm{~m} / \mathrm{sec}$

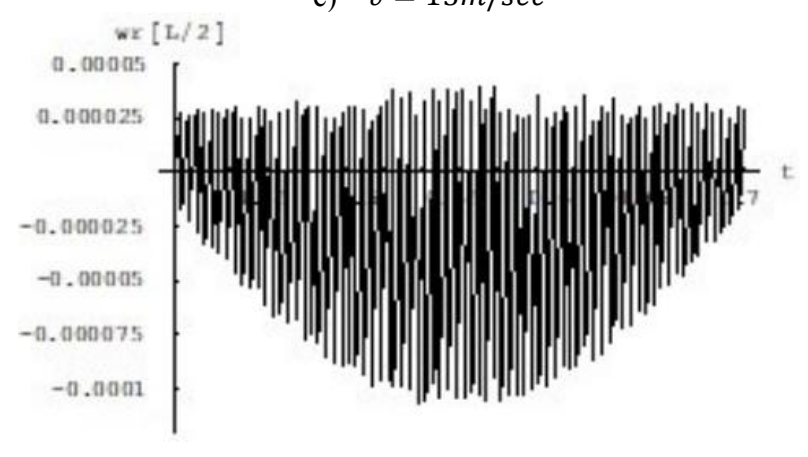

d) $v=20 \mathrm{~m} / \mathrm{sec}$

Figure 29. (Roughness 0,04), w(in m), t(in sec)

The maximum motion of a short bridge due to the roughness alone $(d=0.04 \mathrm{~m})$

for a vehicle moving with different speeds

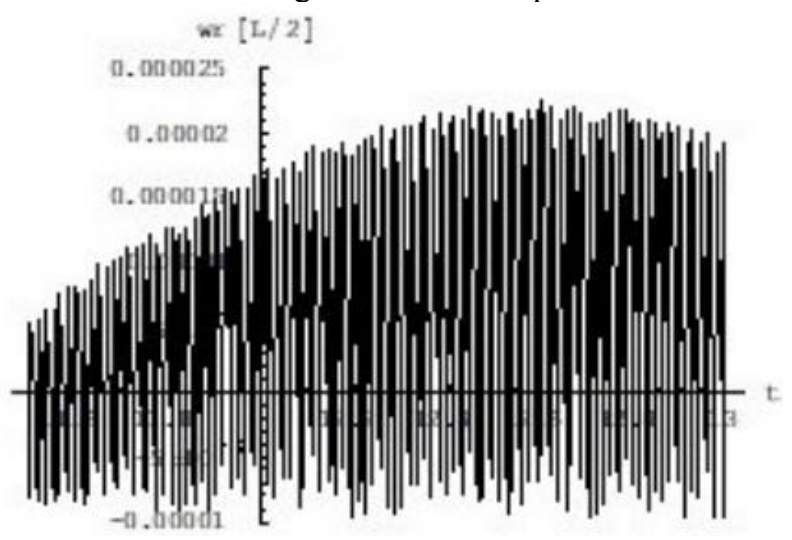

a) $v=5 \mathrm{~m} / \mathrm{sec}$

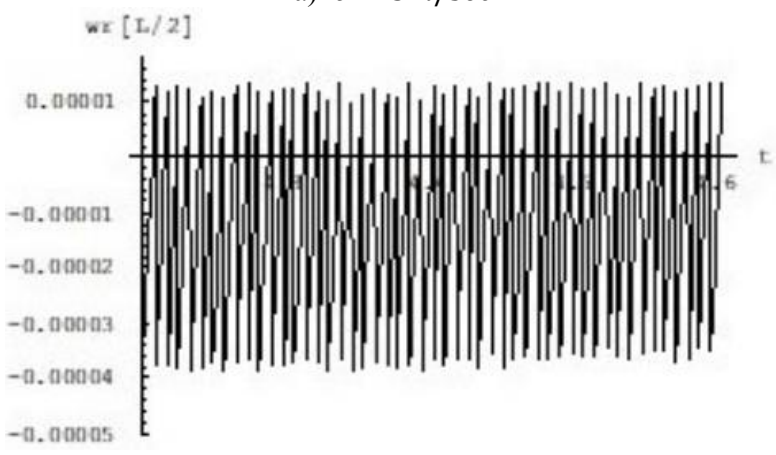

b) $v=10 \mathrm{~m} / \mathrm{sec}$ 


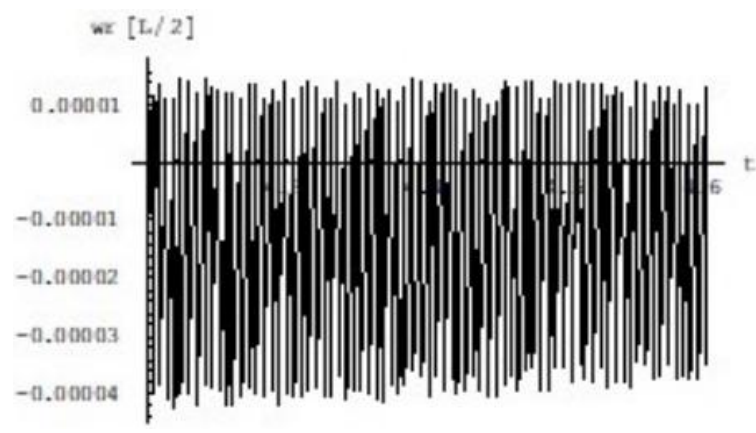

c) $v=15 \mathrm{~m} / \mathrm{sec}$

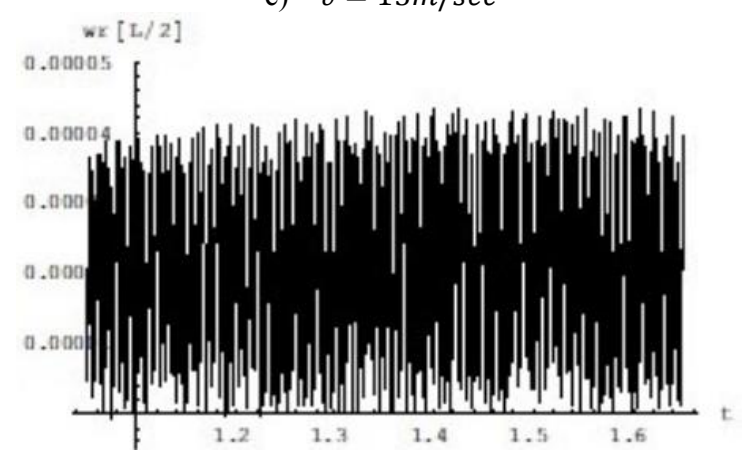

d) $v=20 \mathrm{~m} / \mathrm{sec}$

Figure 30. (Roughness 0,04$)$, w(in $m$ ), $t($ in sec)

The maximum motion of a long bridge due to the roughness alone $(d=0.04 m)$ for a vehicle moving with different speeds.

From the above plots it is obtained the following Table 1.

Table 1. Motion of short or long bridge for different velocities

\begin{tabular}{|c|c|c|c|c|}
\hline & $\begin{array}{c}v= \\
5 \mathrm{~m} / \mathrm{sec}\end{array}$ & $\begin{array}{c}v=10 \mathrm{~m} / \\
\mathrm{sec}\end{array}$ & $\begin{array}{c}v= \\
15 \mathrm{~m} / \mathrm{sec}\end{array}$ & $\begin{array}{c}v=20 \mathrm{~m} / \\
\mathrm{sec}\end{array}$ \\
\hline $\begin{array}{c}\text { Short } \\
\text { Bridge }\end{array}$ & 0.000030 & 0.000080 & 0.000116 & 0.000135 \\
\hline $\begin{array}{c}\text { Long } \\
\text { Bridge }\end{array}$ & 0.000033 & 0.000054 & 0.000057 & 0.000045 \\
\hline
\end{tabular}

The findings of Table 1 can be expressed in diagrams of Figure 31.

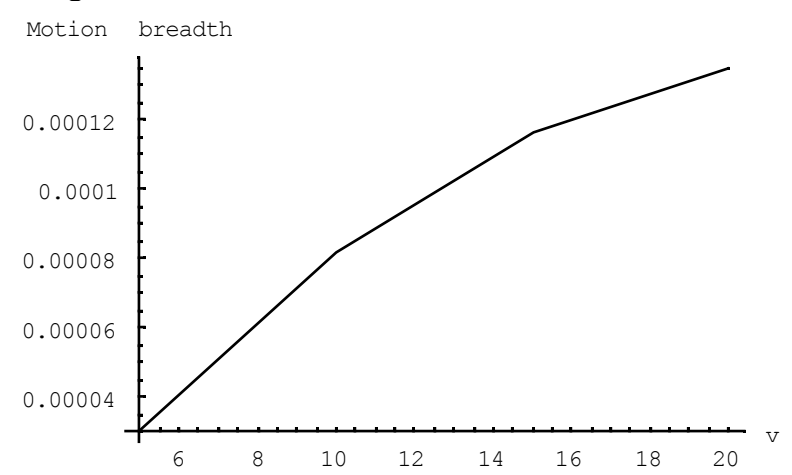

a) Short Bridge

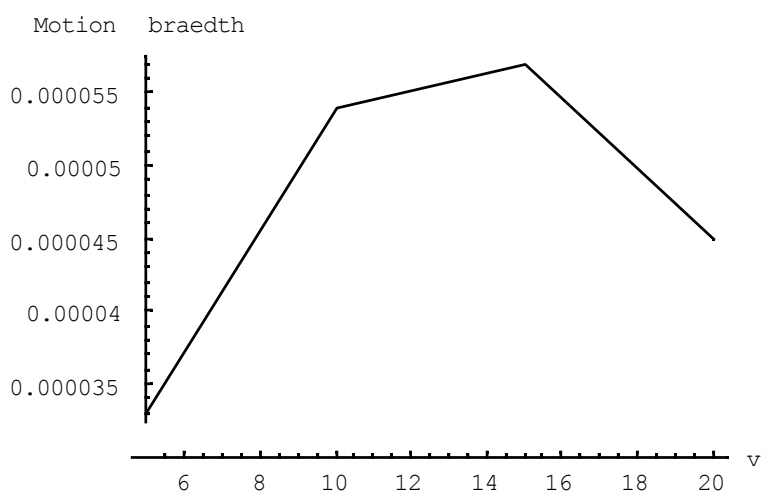

b) Long Bridge

Figure 31. motion breadth (in $\mathrm{m}$ ), velocity (in $\mathrm{m} / \mathrm{sec}$ )

The influence of the speed from the above figures it can be seen that for higher speeds the problem of roughness decreases.

\section{Conclusions}

Considering the results of the above models, the following conclusions can be drawn:

\subsection{Regarding the Irregularities}

a. Following the remarks of paper ${ }^{[21]}$ the length of the irregularities used is $1.00 \mathrm{~m}$.

b. As expected higher irregularities are more effective than lower ones.

c. In both cases of bridges (short and long bridges), the most effective position to build an irregularity is in about the middle length of the bridge.

In particular, on short bridges with light vehicles we observe a reduction of deformations up to $\sim 6,5 \%$ for irregularities built in the middle of the bridge compared to the case of irregularities at the entrance of the bridge.

On short bridges but with big lorries the corresponding reduction of deformations reaches up to $\sim 10 \%$.

On long bridges with short vehicles or big lorries we observe a reduction up to $\sim 2 \%$.

On long bridges with short vehicles it is also possible to observe an increase of deformations instead of a decrease one due to the impact forces (see Figure 17 in relation to Figure 16).

d. Regarding the used vehicle model, we note that for short bridges the real model (model 3) must always be used, as the differences from other models range from $19 \%$ (for light vehicle) to $30 \%$ (for big lorries).

The corresponding differences in long bridges are smaller and range from 0.5 to $2 \%$. Therefore one can even use the model 1. 


\subsection{Regarding the Roughness}

a. The additional motion caused by the roughness even for bad road surfaces is negligible, for both cases of light vehicles and big lorries ranging from 0.2 (smooth road) to $1.5 \%$ (bad road). However in the case of a cobbled road (which is a special case of road between roughness and irregularity) an additional increase of bridge motion of about $10 \%$ is developed.

b. Passengers trouble can be expressed in a number of ways.

The most usual and easy way is to use either the range of motion of the bridge or the acceleration of this motion due to roughness alone, which happens in low periods. In this paper we follow the first way.

An important factor affecting the passenger's trouble is the rapidly changing speed of a vehicle, which moves on the bridge.

From the relevant diagrams of Figures $31 \mathrm{a}$ and $31 \mathrm{~b}$ we observe the following:

On short bridges, higher speeds cause more trouble, but the rate of this trouble augmentation decreases dramatically as the speed increases.

On long bridges the reduction of the trouble is obvious as the speed of the vehicle increases.

\section{References}

[1] Carey W.N. Jr., Irick P.E., "The pavement serviceability-performance", Concept. HRB, Bull. 250, 1960, 40-48.

[2] Kaneshige I. "Power spectra analysis of a vehicle vibration by Road Surface Roughness", J. of Society of Automotive Eng. of Japan, V. 18(4), 1964, 261267.

[3] Kawai T., Morisaki K., "Relationships between Characteristics and Road Surface and Riding Comfort of Vehicle", Mitsubishi Juko Giho, V. 2(2), 1965, 20-27.

[4] Dodds G.J., Robson J.D., "Description of Road Surface Roughness", J. of Sound and Vibration, V. 31(2), 1973, 175-183.

[5] Healey A.J., Nathman E., Smith C.C., "An Analytical and Experimental Study of Automobile Dynamics with Random Roadway Imputs", Journal of Dynamic Systems, Measurement and Control, Series G. Vol. 99, 1977, 284-292.

[6] Honda H., Kajikawa Y., Kobori T., "Spectra of Road Surface Roughness on Bridges", J. of Structural Division (ASCE), V. 108, 1982, 1956-1966.

[7] Wang T.L., Huang D., "Cable-Stayed Bridge vibration due to road surface roughness", Journal of Structural Engineering, 118(5), 1995, 1354-1374.

[8] Cheng Y.S., Au F.T.K., Cheung Y.K., and Zheng D.Y., "On the Separation between Moving Vehicles and Bridge", J. of Sound and Vibration 222(50), 1999, 781-810.

[9] Fatard M., Laflamme M., Savarad M., and Bennur M., "Dynamic Analysis of Existing Continuous Bridge", Journal of Bridge Engineering, 3(1), 1998, 28-37.

[10] Chang D., and Lee H., "Impact factors for simplespan highway girder bridges", Journal of Structural Engineering, 120DeWolf(3), 1994, 704-715.

[11] Huang D.P., and Wang T.L., "Vibration of highway steel bridges with longitudinal grades", Computers and Structures, 69(2), 1998, 235-245.

[12] Kou J.W., and DeWolf J.T., "Vibration behavior of continuous span highway bridge-influencing variables", Journal of Structural Engineering, 123(3), 1997, 333-344.

[13] Yang Y.B., Liao S.S., and Lin B.H., "Impact Formulas for Vehicles Moving over Simple and Continuous Beams", Journal of Structural Engineering, 121(11),1995, 1644-1650.

[14] Wang P., Ding Yong., "Optimazation of vehicleinduced vibration and structure-born noise of Bridge Based on computing", Int. J. of Engineering and Technology, V. 11(3), 2019.

[15] Wang G., Zhou J., "Research on the Influence of Pavement Roughness level on the Driving Comfort", Int. Conference on Civil and Hydraulic Engineering, IOP Conf. Series: Earth and Enviromental Sciences 304, 2019.

DOI: 10. 1088/1755-1315/304/3/032059.

[16] Koc M.A., Esen I., "Modeling and analysis of vehicle-structure road coupled interaction considering structural flexibility, vehicle parameters and road roughness", Journal of Mechanical Science and Technology, 31(5), 2017, 2057-2074.

[17] Park D.W., Papagiannakis A.T., Kim I.T., “Analysis of Dynamic Vehicle loads using Vehicle Pavement interaction model", KSCE Journal of Civil Engineering, 18(7), 2014, 2085-2092.

[18] Yang Y.B., Li Y.B., Chang K.C., "Effect of road surface roughness on the response of a moving vehicle for indentification of Bridge frequencies", Interaction and Multiscale Mechanics, V. 5(4), 2012, 347-368.

[19] Kim C.W., Inone S., Suriura K., "Extracting bridge frequencies from dynamic responses of two passing vehicles", Insights and Innovations in Structural Engineering Mechanics and Computation - Zingoni Ed, 2016, 1858-1860. 
[20] Lalthlamuana R., Talukdar S., "Conditions of Visibility of Bridge Natural Frequency in Vehicle Vertical Acceleration", Procedia Engineering 144, 2016, 26-33.

[21] Michaltsos G.T., Konstantakopoulos T.G., "Dynamic response of a bridge with surface deck irregularities", J. of Vibration and Control, 6, 2000, 667-689.

[22] Michaltsos G.T., " Bouncing of a vehicle on an irregularity - A mathematical model", J. of Vibration and Control, 16(2), 2010, 181-206.

[23] Kounadis A.N., "An efficient and simple approximate technique for solving nonlinear initial and boundary-value problems", Computational Mechanics, 9, 1992, 221-231.

[24] LeNgo-Tran T., Hayashikawa T., Matsumoto T., "Three-dimensional bridge-vehicle interaction analysis of simply supported twin I-girder bridge", J. of Structural Engineering V.54A, 2008, 181-188.

[25] Wang T.L., Huang D., Shahawy M., "Vibration and Impact in Multigirder Steel Bridges", J. of Structural Engineering V. 118(8), 1992, 2222-2238.

\section{Appendix A}

When the solid bodies shown in Figure A1, with angular velocities $\theta_{1}$ and $\theta_{2}$ and rotational inertias $I_{1}$ and $I_{2}$ are collided, after their bouncing, the new angular velocity of the first body after impact is given by:

$\theta_{1 a}=\frac{\left(I_{1}-\varepsilon I_{2}\right) \theta_{1 f}+(1+\varepsilon) I_{2} \theta_{2 f}}{I_{1}+I_{2}}$

where $\varepsilon$ is the percentage of the energy remaining after impact (which for regular wheels is between 0.80 and 0.97 , depending on the inner pressure of the wheel) and $f$ and $\alpha$ express the angular velocities before and after impact.

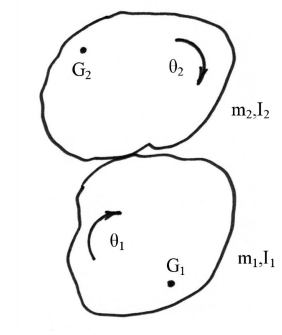

Figure A1

Setting into (A1) $I_{1}=\infty$ and $\theta_{1 f}=0$, we obtain:

$\theta_{2 a}=-\varepsilon \theta_{2 f}=-\varepsilon \theta$

\section{Appendix B}

A body under the action of a moment for a period of time $\Delta t$, acquires a torsional momentum (or impulse) equal to:

$G=M \cdot \Delta t$

It is proved that

$G=I \cdot \omega$

where I the rotational inertia of the body and $\omega$ its angular velocity.

A wheel, has rotational inertia $I=m_{o} i^{2}$ or finally $I=m_{o} R^{2} / 2$

where $m_{0}$ is the mass of the wheel, $i$ its inertia radius and $\mathrm{R}$ the radius of the wheel.

According to appendix A, the impact of a wheel with radius $\mathrm{R}$ on a solid plate is expressed by

$G=M \cdot \Delta t=N \cdot \Delta t \cdot R=I \cdot \omega=m_{o} \varepsilon R^{2} \omega=m_{o} \varepsilon \cdot \frac{R^{2}}{2} \cdot \frac{v}{R}$ and finally

$N=G_{\Delta t \rightarrow 0}=\varepsilon \cdot m_{o} v / 2$

where $\mathrm{N}$ is the force arising from the impact and acting on the common tangent of two solids at the point of impact. 